\title{
New Kohn-Sham density functional based on microscopic nuclear and neutron matter equations of state
}

\author{
M. Baldo* \\ Instituto Nazionale di Fisica Nucleare, Sezione di Catania, Via Santa Sofia 64, I-95123 Catania, Italy \\ L. M. Robledo ${ }^{\dagger}$ \\ Dep. Física Teórica (Módulo 15), Universidad Autónoma de Madrid, E-28049 Madrid, Spain
}

P. Schuck ${ }^{\ddagger}$

Institut de Physique Nucléaire, CNRS, UMR8608, F-91406 Orsay, France, Université Paris-Sud, Orsay F-91505, France, and Laboratoire de Physique et Modélisation des Milieux Condensés, CNRS et Université Joseph Fourier, 25 Avenue des Martyrs, BP 166, F-38042 Grenoble Cedex 9, France

\author{
X. Viñas ${ }^{\S}$ \\ Departament d'Estructura i Constituents de la Matèria and Institut de Ciències del Cosmos, Facultat de Física, \\ Universitat de Barcelona, Diagonal 647, 08028 Barcelona, Spain
}

(Received 27 September 2012; revised manuscript received 20 May 2013; published 10 June 2013)

\begin{abstract}
A new version of the Barcelona-Catania-Paris energy functional is applied to a study of nuclear masses and other properties. The functional is largely based on calculated $a b$ initio nuclear and neutron matter equations of state. Compared to typical Skyrme functionals having 10-12 parameters apart from spin-orbit and pairing terms, the new functional has only 2 or 3 adjusted parameters, fine tuning the nuclear matter binding energy and fixing the surface energy of finite nuclei. An energy rms value of $1.58 \mathrm{MeV}$ is obtained from a fit of these three parameters to the 579 measured masses reported in the Audi and Wapstra [Nucl. Phys. A 729, 337 (2003)] compilation. This rms value compares favorably with the one obtained using other successful mean field theories, which range from 1.5 to 3.0 MeV for optimized Skyrme functionals and 0.7 to 3.0 for the Gogny functionals. The other properties that have been calculated and compared to experiment are nuclear radii, the giant monopole resonance, and spontaneous fission lifetimes.
\end{abstract}

DOI: 10.1103/PhysRevC.87.064305

PACS number(s): 21.60.Jz, 21.30.-x, 21.10.Dr, 21.65.Mn

\section{INTRODUCTION}

The physics of atomic nuclei and nuclear systems is very rich but extremely complicated to describe. It unites many of the toughest aspects of the quantum many body problem in a single spot. To name only a few, we can mention the existence of four different types of fermions (proton/neutron, spin up/down), the relevance of pairing and quartet correlations in the dynamics, or the role of the mean-field approach as a firstorder approximation, while quantum fluctuations are very important as a consequence of the smallness of nuclei. Moreover, the nucleon-nucleon $(N N)$ force is very complicated with its hard core, tensor (dipolar) components, and strong spin-orbit interactions. It is remarkable that nuclear theory has nowadays mastered a great deal of those challenges and many of the experimental data are very well described either on a phenomenological basis, or, more rarely, with microscopic approaches. In this work we try to progress with the latter strategy.

Theories aiming towards a global description of low-energy nuclear data always start with a mean-field description and

\footnotetext{
*baldo@ct.infn.it

†luis.robledo@uam.es

${ }^{\ddagger}$ schuck@ipno.in2p3.fr

${ }^{\S}$ xavier@ecm.ub.es
}

a semiphenomenological nuclear energy density functional. In a recent paper [1], we proposed an approach slightly different from the usual for the establishment of a nuclear energy density functional. We tried to follow as much as possible the Kohn-Sham (KS) [2] density functional theory (DFT), which is based on the Hohenberg-Kohn theorem [3] and is of common use, e.g., in atomic and molecular physic [4-9]. The KS method introduces a set of auxiliary single-particle states and takes for the kinetic energy the Slater form in this basis. In the KS method the energy density functional is written as a sum of two terms. One of them corresponds to the uncorrelated kinetic energy density plus the direct contribution derived from the underlying two-body (Coulomb) interaction. The other piece contains an unknown term for the exchange and correlation energies. For a practical implementation of the method, the latter term has to be guessed and therefore many strategies have been developed over the years to describe different physical phenomena. A popular strategy in atomic and molecular physics is to use accurate theoretical calculations (mainly by Monte Carlo techniques) as a function of the density in simplified models where finite size effects are neglected. The results of those calculations are used to make an educated guess of the unknown exchange and correlation part of the functional in the real system. In this way the minimization procedure gives Hartree-like equations for the single-particle states, where the potential part includes 
in an effective way the overall exchange and correlation contribution.

The application of the KS-DFT scheme to self-bound systems like atomic nuclei is not straightforward, because of the absence of an external confining potential. The usual procedure used with Skyrme functionals has been justified recently [10-12] by reformulating the problem in the "intrinsic" system.

In nuclear physics to build up the exchange and correlation contribution amounts to a detailed determination of the ground state of symmetric nuclear matter and of neutron matter to be used as a guide for finite nuclei energy density functionals. Accurate nuclear matter calculations are very complicated and not as much advanced as for electron systems of condensed matter. Nonetheless, in recent years, quite some progress has been achieved and we build our KS-DFT approach on the equation of state (EOS) of Baldo et al., developed in Refs. [1319], which is based on the well-known hole line expansion. This EOS is calculated from realistic two-body and three-body interactions treated at the Brueckner Hartree-Fock level. It is compatible with phenomenological constraints coming from data of heavy-ion reactions [19], as well as from the analysis of astrophysical observations [20,21]. It is worth mentioning that only two works exist which tried to follow the same strategy as here, one by Fayans [22] and the other by Steiner et al. [23]. However, they are based on a somewhat older EOS [24].

The functional obtained will be used to describe finite nuclei at the mean-field level, concentrating on the binding energy systematic. Therefore, it is important to include beyond-meanfield effects having a strong impact on binding energies and not present in nuclear matter. The most obvious one is the correlation energy associated with symmetry restoration that will be accounted for in the case of translational and rotational invariance.

A different approach to nuclear energy density functional (EDF) was developed on the basis of the chiral two- and three-body forces [25-29]. The final form of the EDF contains a set of contact interactions, similar to the Skyrme functionals, with a corresponding set of about a dozen of parameters. This part is supplemented by a contribution coming from pion exchanges, which is nonlocal and treated through the density matrix expansion procedure. Preliminary calculations [29] on a selected set of nuclei show a slight improvement with respect to the standard Skyrme functionals and open a promising prospect for large-scale fitting procedures.

The reason for such an intense focus on an accurate theoretical description of bulk properties of nuclei all over the nuclide chart is discussed, for instance, in Ref. [30]: Many physical scenarios involve neutron-rich nuclei which are far from reach from an experimental point of view and therefore reliable theoretical predictions are the only possible option at present. The present status is that good agreement between different theoretical models is to be expected as long as we do not move towards the neutron drip line, a region of relevance in stellar nucleosynthesis processes. Therefore, a new model based on somewhat different ideas closer to the KS-DFT approach than the more traditional Skyrme-like EDF (inspired by contact central potentials) can help to clarify the uncertainties associated with present-day models.
In the present work, a KS approach based on the same microscopic bulk input as in Ref. [1] is used for the particlehole channel. However, we are able to reduce the number of parameters by two without losing accuracy. As before, we consider an additional bulk parameter for a precise adjustment of the numerically obtained $E / A$ value. However, for the surface, we reduce the number of parameters from three to one by the condition that, in infinite symmetric and infinite pure neutron matter, the strength parameters of the finite range term reproduce the coefficients of the quadratic terms of the bulk energy density obtained from the microscopic calculation. Therefore, no subtraction term in the finite range part as in Ref. [1] is necessary. The range $r_{0}$ of the surface term remains the only adjustable parameter; see the more detailed discussion below. Although there are, together with the strength of the spin-orbit term, three parameters in the particle-hole channel, we want to emphasize that the most relevant are the ones from bulk and surface. We stress this point, because it seems to us a reduction to two basic physical inputs to the binding energy of nuclei: energy per particle of infinite matter and surface energy. That this is possible is as much surprising as it is gratifying. It also should be mentioned that the adjustment of those two parameters is extremely sensitive: Both have to be fine tuned to the order of $10^{-3}$. This sensitivity points to a well-defined physical content of the parameters.

It should be pointed out, however, that KS-DFT addresses only the ground state and is, in principle, not tailored to describe excited states of the system. Nevertheless, we also use it for the description of the giant monopole resonances (GMRs).

The paper is organized as follows. The following section is devoted to briefly recalling our previous Barcelona-CataniaParis (BCP) functional. In the third section, the new EDF built up in this paper is discussed. The results obtained with this improved functional, which we call BCPM (BarcelonaCatania-Paris-Madrid), are also presented in the same section. The predictive power of the BCPM functional regarding other observable such as charge radii, quadrupole, and octupole deformations and fission barriers are discussed in the fourth section. The ability of the BCPM functional for describing the isoscalar GMR is the subject of the fifth section. Finally, the summary and conclusions are given in the last section.

\section{FORMER BCP FUNCTIONAL}

The former BCP functional was proposed in Ref. [1]. This and subsequent refinements [31] are based on the Kohn-Sham density functional theory (KS-DFT), where the one body density $\rho(\mathbf{r})$ plays a central role. In the KS-DFT theory an auxiliary set of $A$ orthonormal wave functions $\varphi_{i}(\mathbf{r})$, where $A$ is the mass number, is introduced to express formally the density as if it were obtained from a Slater determinant as a sum of the product of single-particle wave functions,

$$
\rho(\mathbf{r})=\sum_{i}\left|\varphi_{i}(\mathbf{r})\right|^{2},
$$

with the $\varphi$ 's determined from the minimization of the groundstate energy. In condensed matter and atomic physics the EDF 
$E[\rho(\mathbf{r})]$ is usually split into two parts [2]:

$$
E=T_{0}[\rho]+W[\rho] .
$$

The first piece $T_{0}[\rho]$ corresponds to the uncorrelated kinetic energy written in the usual manner as

$$
T_{0}=\frac{\hbar^{2}}{2 m} \sum_{i} \int d \mathbf{r}\left|\nabla \varphi_{i}(\mathbf{r})\right|^{2} .
$$

The other piece, $W[\rho]$, contains the potential energy and the correlated part of the kinetic energy. In the BCP family of functionals this part is given as the sum of spin-orbit energy, the Coulomb part, and the nuclear energy term, which depends on the neutron and proton densities $\rho_{n}$ and $\rho_{p}$, respectively. In nuclear physics, contrary to the situation in condensed matter and atomic physics, the contribution of the spin-orbit interaction to the energy functional is very important. Nonlocal contributions have been included in DFT in several ways long ago (see Ref. [32] for a review of this topic). In line with this, we split the spin-orbit part into an uncorrelated part $E^{\text {s.o. }}$ plus a remainder not treated explicitly here. The form of the uncorrelated spin-orbit part is taken exactly as in typical Skyrme [33,34] or Gogny forces [35,36]. Another piece that we explicitly split off is the Coulomb part $E_{C}$. We treat this contribution at lowest order, i.e., the direct term plus the exchange contribution in the Slater approximation, that is,

$$
E_{C}^{H}=\frac{1}{2} \iint d \mathbf{r} d \mathbf{r}^{\prime} \rho_{p}(\mathbf{r})\left|\mathbf{r}-\mathbf{r}^{\prime}\right|^{-1} \rho_{p}\left(\mathbf{r}^{\prime}\right),
$$

and

$$
E_{C}^{\mathrm{ex}}=-\frac{3}{4}\left(\frac{3}{\pi}\right)^{1 / 3} \int d \mathbf{r} \rho_{p}(\mathbf{r})^{4 / 3},
$$

with $E_{C}=E_{C}^{H}+E_{C}^{\mathrm{ex}}$.

The nuclear energy functional contribution $E_{\text {int }}\left[\rho_{n}, \rho_{p}\right]$ contains the nuclear potential energy, as well as additional correlations. This contribution is divided into a finite range term $E_{\text {int }}^{\mathrm{FR}}\left[\rho_{n}, \rho_{p}\right]$ to account for correct surface properties and a bulk correlation part $E_{\text {int }}^{\infty}\left[\rho_{n}, \rho_{p}\right]$ that we take from one of the most advanced microscopically determined EOS existing so far in the literature [13-19], as mentioned before. Collecting all these contributions our final KS-DFT functional reads

$$
E=T_{0}+E^{\text {s.o. }}+E_{\mathrm{int}}^{\infty}+E_{\mathrm{int}}^{\mathrm{FR}}+E_{C} .
$$

The functional is supplemented by a density-dependent pairing interaction and some beyond-mean-field corrections (see below).

One of the prominent results of our recent work within the KS-DFT BCP density functional scheme [1] was that, with a very reduced number of adjustable parameters to finite nuclei, rms values for binding energies and radii came out to be of the same quality as the ones of some Skyrme [33] or Gogny [36] functionals. The reduction of the number of parameters stemmed from the fact that, prior to a fit of the functional to nuclear masses, the bulk part of the functional $E_{\text {int }}^{\infty}$ was adjusted to the microscopic EOS obtained in Refs. [13-19] by fitting polynomials in the total density $\rho=\rho_{n}+\rho_{p}$ to microscopic results in symmetric and pure neutron matter, followed by a quadratic interpolation in the asymmetry parameter $\beta=$ $\left(\rho_{n}-\rho_{p}\right) / \rho$ between these two limits.

The curve obtained in the polynomial fit can be slightly changed without changing significantly the mean square deviation to the microscopic EOS. However, although the microscopic EOS is state of the art, the uncertainties in the underlying interaction and the ones attributed to the approximations involved translate to minor uncertainties in the computed points of the microscopic EOS that will eventually lead to minor changes in the polynomial curve. We exploit this limited freedom to produce polynomial fits with slightly different values of $E / A$ at saturation (around $E / A=16 \mathrm{MeV})$. The resulting EDFs are then used in the finite nuclei fit (see below) and the one leading to the smallest binding energy rms is chosen. Only changes of the order of a few hundred $\mathrm{KeV}$ in $E / A$ are considered.

The additional surface term in Ref. [1] was of the form

$$
\begin{aligned}
E_{\mathrm{int}}^{\mathrm{FR}}\left[\rho_{n}, \rho_{p}\right]= & \frac{1}{2} \sum_{t, t^{\prime}} \iint d \mathbf{r} d \mathbf{r}^{\prime} \rho_{t}(\mathbf{r}) v_{t, t^{\prime}}\left(\mathbf{r}-\mathbf{r}^{\prime}\right) \rho_{t^{\prime}}\left(\mathbf{r}^{\prime}\right) \\
& -\frac{1}{2} \sum_{t, t^{\prime}} \gamma_{t, t^{\prime}} \int d \mathbf{r} \rho_{t}(\mathbf{r}) \rho_{t^{\prime}}(\mathbf{r})
\end{aligned}
$$

where the index $t$ is the label for neutron and proton, i.e., $t=n, p$, and $\gamma_{t, t^{\prime}}$ is the volume integral of $v_{t, t^{\prime}}(r)$. The subtraction term was introduced not to contaminate the bulk part, determined from the microscopic infinite matter calculation. For the finite range form factor $v_{t, t^{\prime}}(r)$ a simple Gaussian ansatz,

$$
v_{t, t^{\prime}}(r)=V_{t, t^{\prime}} e^{-r^{2} / r_{0}^{2}},
$$

was made. The strength parameters were chosen to distinguish only between like and unlike particles, i.e.,

$$
V_{p, p}=V_{n, n}=V_{L} ; \quad V_{n, p}=V_{p, n}=V_{U},
$$

so that the finite range term contained three adjustable parameters: $V_{L}, V_{U}$, and $r_{0}$. Together with the adjustment of the bulk and the spin-orbit strength, the functional in Ref. [1] has, all in all, five adjustable parameters, even though they are not all to be considered on the same level of significance. We come back to these considerations later in the text where we also show how to avoid the subtraction term in the finite range part of the energy.

To describe properly open-shell nuclei one has to introduce pairing correlations. The formal way of including pairing within the DFT is also known from the generalization of the Hohenberg-Kohn theorem to paired systems [37]. In Ref. [1] we have introduced pairing in our functional through the BCS approach using a zero-range density-dependent interaction adjusted to reproduce the neutron gaps of the Gogny force in symmetric nuclear matter with $m^{*}=m$ [38].

The free parameters of the EDF reported in Ref. [1] were fitted either to reproduce the binding energies of a few spherical nuclei or binding energies and charge radii of the same set of spherical nuclei. We called these functionals $\mathrm{BCP} 1$ or $\mathrm{BCP} 2$, respectively. The predictive power of these functionals was illustrated by computing the binding energies and charge radii of 161 spherical nuclei between ${ }^{16} \mathrm{Ne}$ and ${ }^{224} \mathrm{U}$. We found that the rms in energy and charge radii are similar to the ones 
obtained with successful mean-field models such as the Gogny D1S force [36], the Skyrme SLy4 interaction [33], and the relativistic mean-field parametrization NL3 [39].

In subsequent publications we explored the properties of BCP1 and BCP2 in describing quadrupole and octupole deformation ground-state properties, fission, excited octupole states, etc. [40-42]. In dealing with deformed nuclei the most relevant beyond-mean-field effect, namely the rotational energy correction has been considered in an approximate way [43]. Also some other parametrizations of the functional based on fitting to the binding energy of deformed nuclei instead of the fitting to spherical nuclei have been considered [31].

We would like to point out that for other EDFs, like the ones of the Skyrme, Gogny, or relativistic mean-field type, the number of parameters seems much higher, typically more than ten. However, many of those parameters are implicitly used to get a reasonable nuclear matter and neutron matter EOS. The advantage of our KS-DFT procedure is that one clearly separates the tasks of reproducing the nuclear matter EOS from the most prominent finite size effects, namely the surface and Coulomb energies and the spin-orbit potential.

\section{IMPROVEMENTS OVER BCP AND RESULTS}

\section{A. New polynomial fitting}

Following Refs. [1,31] we write the bulk part of the EDF as

$$
E_{\text {int }}^{\infty}\left[\rho_{p}, \rho_{n}\right]=\int d \mathbf{r}\left[P_{s}(\rho)\left(1-\beta^{2}\right)+P_{n}(\rho) \beta^{2}\right] \rho,
$$

where the interpolating polynomials for symmetric and pure neutron matter, $P_{s}(\rho)$ and $P_{n}(\rho)$, respectively, read

$$
P_{s}(\rho)=\sum_{n=1}^{5} a_{n}\left(\frac{\rho}{\rho_{0}}\right)^{n}, \quad P_{n}(\rho)=\sum_{n=1}^{5} b_{n}\left(\frac{\rho}{\rho_{0 n}}\right)^{n},
$$

where the reference densities are $\rho_{0}=0.16 \mathrm{fm}^{-3}$ and $\rho_{0 n}=$ $0.155 \mathrm{fm}^{-3}$, respectively.

As compared to the former version of the BCP EDF, the fit of the microscopic EOS has been redone to avoid some rather strong oscillatory behavior in the density-dependent incompressibility of the nuclear matter (see Sec. IV below) when plotted as a function of density. To cure this unwanted effect, the number of theoretical points is increased and a fifth-order polynomial in the density is chosen for fitting the microscopic EOS in symmetric nuclear matter in a wider range of densities, up to $0.625 \mathrm{fm}^{-3}$, instead of the prescription given in Ref. [1]; see also Ref. [31]. Furthermore, special care has been paid to the smoothness not only of the fitted EOS but also of the density-dependent incompressibility. This has been achieved by considering only polynomial fits with smooth high order derivatives with respect to the density.

In Fig. 1 the microscopic EOS for nuclear and neutron matter as well as the corresponding polynomial fits are shown as functions of the density. In the bottom panel, the symmetry energy computed using the polynomial fits as a function of the density is also displayed. As discussed in Ref. [44], the low-density behavior of the microscopic nuclear matter EOS
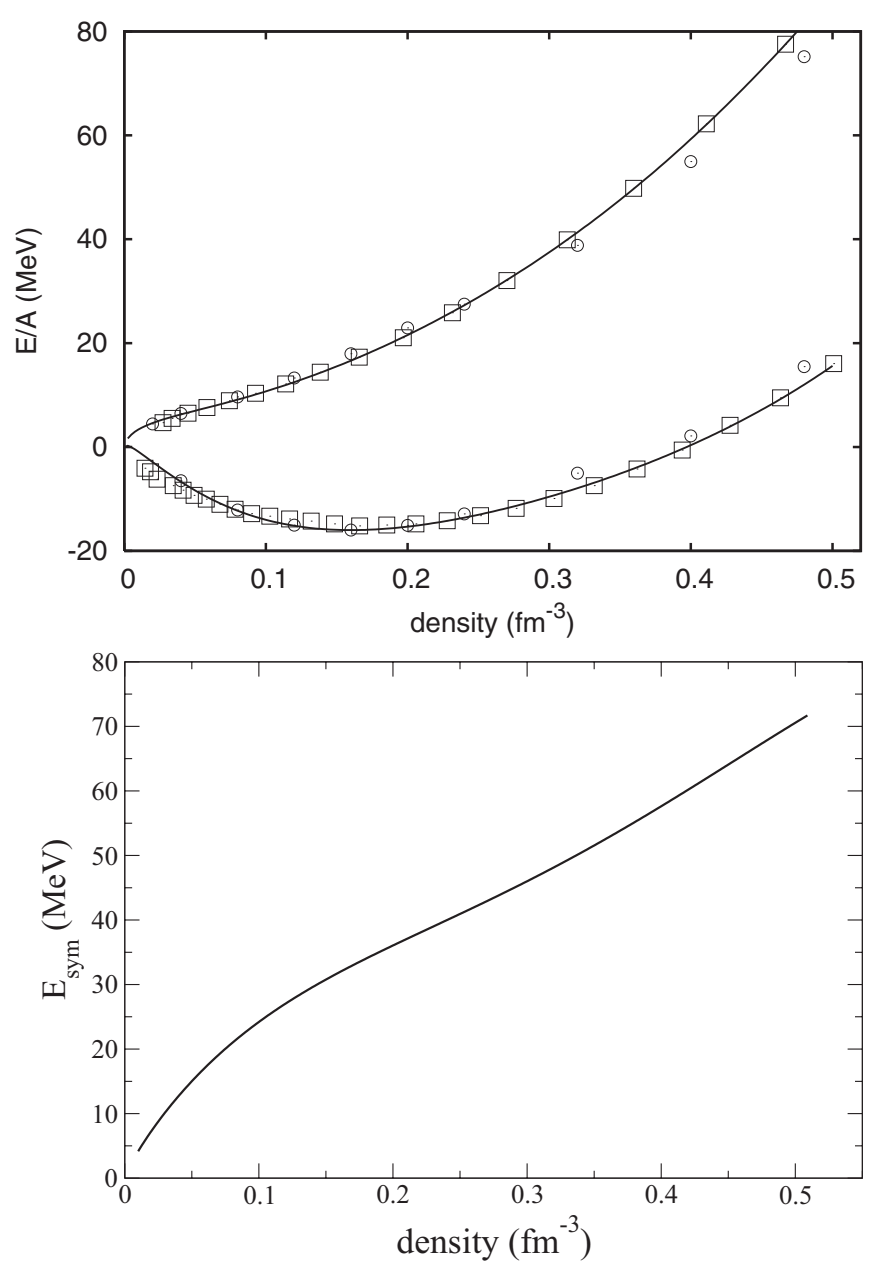

FIG. 1. (Top) EOS of symmetric and neutron matter obtained by the microscopic calculation (squares) and the corresponding polynomial fits (solid lines). For comparison the microscopic EOS of Ref. [45] are also displayed by open circles. (Bottom) Symmetry energy obtained from the polynomial fits.

has a characteristic trend, usually not reproduced by Skyrme and Gogny functionals (see also Refs. [18,31]), missing there quite a substantial part of binding.

Because we want to construct the KS-DFT functional on the basis of the microscopic calculations, the bulk part $E_{\text {int }}^{\infty}$ of the functional, directly related to a realistic EOS, is determined once and for all as in Eq. (3), together with the local density approximation. However, as mentioned before, slightly different polynomial interpolations with different values of $E / A$ at saturation have been considered to improve the finite nuclei results for the binding energies. The saturation density has been kept fixed at the nominal value of $0.16 \mathrm{fm}^{-3}$. For $E / A$ at saturation we have explored the interval between 15.97 and $16.03 \mathrm{MeV}$ in steps of $0.01 \mathrm{MeV}$. For each value of $E / A$ the complete fitting process for finite nuclei has been performed (see below). The optimal value for the saturation energy per particle turns out to be $15.98 \mathrm{MeV}$. The fine tuning of $E / A$ at saturation is to be expected because of the extreme sensitivity of the mass of heavy nuclei to this parameter. In addition, as the fine tuning involves finite nuclei calculations where the surface 
TABLE I. Parameters (in MeV) of the polynomial fits, $P_{s}(\rho)=$ $\sum_{n} a_{n}\left(\rho / \rho_{0}\right)^{n}$ and $P_{n}(\rho)=\sum_{n} b_{n}\left(\rho / \rho_{0 n}\right)^{n}$, for symmetric and neutron matter, respectively. The reference densities are $\rho_{0}=0.16 \mathrm{fm}^{-3}$ and $\rho_{0 n}=0.155 \mathrm{fm}^{-3}$. Two sets of parameters for symmetric matter are given; they correspond to EOS with a minimum at the given values of $E / A$. The parameters are given with six digits, enough to obtain binding energies with an accuracy of a couple of $\mathrm{eV}$. The value $\hbar^{2} c^{2} / 2 M=20.75 \mathrm{MeV}$ has been used.

\begin{tabular}{lrrr}
\hline \hline$n$ & \multicolumn{1}{c}{$b_{n}$} & $a_{n}(E / A=16)$ & $a_{n}(E / A=15.98)$ \\
\hline 1 & -34.972615 & -73.292026 & -73.382673 \\
2 & 22.182307 & 49.964905 & 50.297798 \\
3 & -7.151756 & -18.037601 & -18.366734 \\
4 & 1.790874 & 3.486176 & 3.608359 \\
5 & -0.169591 & -0.243552 & -0.258847 \\
\hline \hline
\end{tabular}

term is included, it can be considered as a way to account for the coupling of volume and surface in finite nuclei.

The values of the coefficients of the fitted polynomials for neutron matter and for two choices of symmetric matter leading to slightly different values of $E / A$ are reported in Table I. A nice feature of the coefficients $a_{n}$ is that they follow a perfect linear dependence when plotted as a function of $E / A$ and therefore the two sets of $a_{n}$ values are enough to reproduce the whole range of $E / A$ values considered with only one parameter.

As an aside, it may be important to mention that the densitydependent part of the functional depends on integer powers of the density only. This is advantageous when trying to overcome the self-energy problem that plagues the use of theories beyond the mean field based on standard nuclear EDFs [46,47].

\section{B. Infinite matter properties}

The pressure $P$ and density-dependent incompressibility $K$ in asymmetric nuclear matter are defined in terms of the energy density in asymmetric nuclear matter $\mathcal{H}(\rho, \beta)$ as [48]

$$
P=\rho^{2} \frac{\partial(\mathcal{H} / \rho)}{\partial \rho}=\rho \frac{\partial \mathcal{H}}{\partial \rho}-\mathcal{H}
$$

and

$$
K=9 \rho \frac{\partial^{2} \mathcal{H}}{\partial \rho^{2}}=9 \rho^{2} \frac{\partial^{2}(\mathcal{H} / \rho)}{\partial \rho^{2}}+\frac{18}{\rho} P .
$$

For symmetric matter $(\beta=0)$ at saturation density the $K(\rho)$ just defined reduces to the well-known incompressibility modulus $K_{0}=\left.9 \rho^{2} \frac{\partial^{2}(\mathcal{H} / \rho)}{\partial \rho^{2}}\right|_{\rho=\rho_{0}}$, where $\rho_{0}$ is the saturation density. For later use, it will be convenient to introduce the coefficient $K^{\prime}$ connected to the so-called skewness coefficient [49] by

$$
K^{\prime}=-Q=-\left.27 \rho^{3} \frac{\partial^{3}(\mathcal{H} / \rho)}{\partial \rho^{3}}\right|_{\rho=\rho_{0}}
$$

Other relevant quantities in asymmetric nuclear matter are the symmetry energy and its first and second derivatives with respect to the density which govern the isovector part of the
TABLE II. Infinite nuclear matter properties of BCPM. All the parameters, except $\rho_{0}$ (in $\mathrm{fm}^{-3}$ ) and the dimensionless effective mass $m / m^{*}$, are given in $\mathrm{MeV}$

\begin{tabular}{lccccccc}
\hline \hline$B / A$ & $\rho_{0}$ & $m / m^{*}$ & $J$ & $L$ & $K_{0}$ & $K^{\prime}$ & $K_{\text {sym }}$ \\
\hline-15.98 & 0.16 & 1.00 & 31.90 & 52.96 & 212.4 & 879.6 & -96.75 \\
\hline \hline
\end{tabular}

nuclear interaction. The symmetry energy is defined as

$$
E_{\mathrm{sym}}(\rho)=\left.\frac{1}{2} \frac{\partial^{2}}{\partial \beta^{2}}\left(\frac{\mathcal{H}}{\rho}\right)\right|_{\beta=0} .
$$

At saturation density one defines the symmetry energy coefficient as $J=E_{\text {sym }}\left(\rho_{0}\right)$ and two coefficients more, $L$ and $K_{\text {sym }}$, directly related to the first and second derivatives of Eq. (8) with respect to the density at saturation, respectively,

$$
L=\left.3 \rho \frac{\partial E_{\text {sym }}}{\partial \rho}\right|_{\rho=\rho_{0}}, \quad K_{\text {sym }}=\left.9 \rho^{2} \frac{\partial^{2} E_{\text {sym }}}{\partial \rho^{2}}\right|_{\rho=\rho_{0}} .
$$

The values of the incompressibility modulus $K_{0}$ and the coefficient $K^{\prime}$ defined in symmetric nuclear matter as well as the coefficients $J, L$, and $K_{\text {sym }}$, which are related to the symmetry energy, are displayed in Table II.

The binding energy and the saturation density in nuclear matter are constrained by nuclear masses and electron scattering experimental data. The range of accepted values of the incompressibility modulus $K_{0}$ is constrained by the experimental excitation energies of the isoscalar GMR in finite nuclei since the pioneering works of Bohigas and collaborators [50] and Blaizot [51]. However, different estimates of $K_{0}$ using different mean-field models predict slightly different values for this coefficient. Recently, a value $K_{0}=230 \pm 30 \mathrm{MeV}$ has been proposed [52] as a compromise among the different available estimates. The value of the symmetry energy coefficient $J$, which dictates the isospin dependence of the nuclear interaction, is constrained by experimental data on heavy-ion collisions, pigmy dipole resonances, and analog states. A range $30 \leqslant J \leqslant 35 \mathrm{MeV}$ has been recently proposed for this coefficient [53]. The density content of the symmetry energy is a relevant physical quantity related with many phenomena not only in terrestrial nuclei but also in neutron stars. Since the celebrated correlation established by Brown [54] between the slope of the symmetry energy and the neutron skin in ${ }^{208} \mathrm{~Pb}$, a considerable effort has been devoted to constrain the $L$ parameter from available data. Antiprotonic atoms, nuclear masses, heavy-ion collisions, giant and pigmy dipole resonances, proton-nucleus scattering, as well as theoretical calculations using a microscopic interactions have been used to extract the $L$ coefficient (see Ref. [55] and references therein). From a compilation of the existing data the range of values $L=55 \pm 25 \mathrm{MeV}$ has been proposed [55,56]. The other two coefficients that characterize asymmetric nuclear matter around saturation, namely $K^{\prime}$ and $K_{\text {sym }}$, are more uncertain. An estimate $K^{\prime}=700 \pm 500 \mathrm{MeV}$ has been proposed in Ref. [52]. The curvature of the symmetry energy, $K_{\text {sym }}$, can be inferred from some nonrelativistic [57] and relativistic [58] calculations in nuclear matter. From these results a range $-200 \leqslant K_{\text {sym }} \leqslant 150 \mathrm{MeV}$ can be inferred. As can be seen 
from a direct inspection of Table II, the nuclear matter properties of our BCPM EDF lie within the accepted ranges of values of the different nuclear matter quantities.

\section{Surface term strengths derived from nuclear matter data and spin-orbit contribution}

In the new version of the functional the Gaussian form factors in the surface term are free to have different ranges depending on the isospin channel, $r_{0 L}$ and $r_{0 U}$, introducing in this way a new parameter as compared to the old BCP1. However, the strengths $V_{L}$ and $V_{U}$ are now no longer considered as free parameters. They have been determined in such a way that the bulk limit of the surface term in Eq. (2), that is,

$$
\frac{1}{2} \sum_{t, t^{\prime}} \gamma_{t, t^{\prime}} \int d^{3} r \rho_{t}(\mathbf{r}) \rho_{t^{\prime}}(\mathbf{r}),
$$

reproduces the $\rho^{2}$ term of the bulk part of the energy density in Eq. (3). Therefore, no subtraction as in Eq. (2) is required. This procedure imposes the following relationships between the surface term strengths and the coefficients $a_{1}$ and $b_{1}$ of the polynomial fits of symmetric and neutron matter, respectively:

$$
\begin{aligned}
& V_{L}=\frac{2 \tilde{b}_{1}}{\pi^{3 / 2} r_{0 L}^{3} \rho_{0}}, \\
& V_{U}=\frac{4 a_{1}-2 \tilde{b}_{1}}{\pi^{3 / 2} r_{0 U}^{3} \rho_{0}} .
\end{aligned}
$$

Here $r_{0 L}$ and $r_{0 U}$ are the ranges of the Gaussian in the surface term, as defined above. The parameter $\tilde{b}_{1}$, defined as $\tilde{b}_{1}=b_{1} \frac{\rho_{0}}{\rho_{0 n}}$, has also been introduced to take into account the different reference densities for symmetric and neutron matter.

However, the spin-orbit strength does not need to be adjusted because the final result is nearly independent of it. The spin-orbit strength is fixed by the requirement to reproduce the magic numbers and therefore depends on the major shells' energy separation. The latter is, roughly speaking, inversely proportional to the effective mass. The same dependence with the effective mass should hold for the spin-orbit strength. Therefore, we take the value $W_{L S}=90.5 \mathrm{MeV}$, which is consistent with BCPM's effective mass of one and the spinorbit strength $W_{L S}=130 \mathrm{MeV}$ of the Gogny force $\left(m^{*}=\right.$ $0.7 \mathrm{~m})$.

From the above discussion we conclude that the number of parameters in the surface term gets reduced from three to two, namely the $r_{0 L}$ and $r_{0 U}$ ranges. As mentioned, the spin-orbit strength $W_{L S}$ is practically fixed from the beginning. However, as we see below, the actual fit allows for $r_{0 L}=r_{0 U}=r_{0}$ and, thus, one is left with one parameter only.

\section{Fitting protocol for finite nuclei}

Owing to the simplicity of the BCP EDF we are in the position to perform mass table evaluations on a personal computer in a reasonable amount of time (a few hours). Therefore, we have decided to include in the fit not only the masses of spherical nuclei but also the ones corresponding to deformed ground states. The only limitation has been the restriction to even-even nuclei. In the fit we have searched for the values of the parameters that minimize the rms deviation for the binding energies,

$$
\sigma^{2}(E)=\frac{1}{N} \sum_{i=1}^{N}\left[B_{\mathrm{th}}(i)-B_{\exp }(i)\right]^{2},
$$

where the sum runs over the set of 579 even-even nuclei with known experimental binding energies, as given in the 2003 evaluation of Audi and Wapstra [59]. The 193 extrapolated binding energies included in Audi and Wapstra's work are excluded from our analysis, although we use them to explore the quality of our fit. Finite nuclei properties are computed within the mean-field framework provided by the well-known Hartree-Fock-Bogoliubov (HFB) method [60]. The pairing interaction is the same as in the original formulation of the functional [1] but using the HFB instead of the BCS method.

\section{E. Details of the HFB calculations}

The HFB calculations have been restricted to axially symmetric solutions. The quasiparticle operators of the Bogoliubov canonical transformation have been expanded in a harmonic oscillator basis with a size (the number of shells) depending on the nuclei considered. For nuclei with $Z<50$ we take a basis with eleven harmonic oscillator shells, for $50<Z<82$ we consider 13 shells and for $Z>82$ a 15 -shell basis is used. These bases are used in a set of constrained calculations providing the potential energy as a function of the quadrupole moment for each nucleus. The minima of the potential energy are used as starting wave functions for an unrestricted minimization. Once the minimum energy solution is found for each nucleus, an extrapolation to an infinite basis is performed in the spirit of Ref. [61]: The HFB equation is solved with an enlarged basis containing two extra shells and the infinite basis energy is obtained by the extrapolation formula $E(\infty)=2 E(N+2)-E(N)(N$ is the initial number of shells).

For the solution of the nonlinear HFB equation, an approximate second-order gradient method is used [62]. The information about the energy curvature reduces the number of iterations substantially as compared to other methods. As it is customary in this kind of calculations, the Coulomb exchange contribution is computed in the Slater approximation [63] and the Coulomb antipairing effect is not explicitly considered (see Ref. [64] for a discussion of this issue). The two-body kinetic energy correction, which is typically considered as a way to correct for the lack of translational invariance of the whole procedure, has been taken into account with the pocket formula of Refs. [65,66] as in previous versions of the BCP functional. In deformed nuclei the correlation energy stemming from symmetry restoration of the rotational invariance (the rotational energy correction) plays a relevant role. It rapidly changes from magic or semimagic nuclei, where it is zero or very small, to strongly deformed midshell nuclei, where it reaches values that can be as large as 6 or $7 \mathrm{MeV}$ in heavy nuclei. This is a consequence of its almost linear dependence with quadrupole deformation and 
therefore its inclusion is relevant to describe masses all over the nuclide chart. The correct way to compute this quantity is by evaluating the angular momentum projected energy associated with each intrinsic state. This is a tremendous task that can fortunately be alleviated by considering an approximation to the projected energy that is obtained in the spirit of the topological Gaussian overlap approximation. This fully microscopic formula, which is similar to the rotational energy correction, can be easily evaluated at the mean-field level and does not involve any phenomenological parameter (see Ref. [43] for details). Finally, as already mentioned, for the pairing part we have taken the density-dependent zero range force of Ref. [38] that was devised to mimic the behavior of Gogny D1 pairing gap in nuclear matter. We have taken care of our effective mass equal to one by renormalizing the pairing force strength given in Ref. [38].

\section{F. Determination of parameters}

The three initial free parameters (i.e., the two ranges and spin-orbit) were at first considered in the fit but soon it became clear that the $E / A$ value given by the polynomial fit should also be taken into account as a free parameter in the way we discussed above. Of the four, it turns out that $\sigma(E)$ has a very smooth dependency on $W_{L S}$ and the minimum value of $\sigma(E)$ was always obtained for spin-orbit strength values around $90 \mathrm{MeV} \mathrm{\textrm {fm } ^ { 5 }}$. As explained before, this value is consistent with the value of $130 \mathrm{MeV} \mathrm{fm}^{5}$ used in Gogny D1S (the difference is related to the different effective masses, which are $m^{*}=m$ for BCP and $m^{*}=0.7 m$ for Gogny D1S and therefore $0.7 \times 130 \approx 90$ ). Another relevant observation is that the binding energy difference $\Delta B=B_{\text {th }}-B_{\text {exp }}$ shows a linear dependence with mass number $A$ (which is sometimes masked by large fluctuations at low A), with a slope that is intimately related to the value of $E / A$ for the bulk. It is almost zero for $E / A \approx 15.97$ and is clearly different from zero and positive for $E / A=16.03$, as can be observed in Fig. 2. It turns out that the value $E / A=15.98$ yields the lowest $\sigma(E)$ value. The final relevant observation is that $\sigma(E)$ depends sensitively on the values of $r_{0 L}$ and $r_{0 U}$ and an accuracy of one part in $5 \times 10^{3}$ is required to obtain reasonable values for that quantity. Systematic explorations with a reduced set of spherical nuclei show that there are two sets of $r_{0 L}$ and $r_{O U}$ values that lead to reasonable values of $\sigma(E)$, namely $r_{0 L}$ equal to $r_{0 U}$ with values around $0.660 \mathrm{fm}$ and $r_{0 L}$ taking values around $0.490 \mathrm{fm}$ and $r_{0 U}$ around $1.050 \mathrm{fm}$. In the latter case, the value of $r_{0 U}$ is more critical than the $r_{0 L}$ value and it has to be kept at the value $r_{0 U}=1.046 \mathrm{fm}$, leaving only one free parameter to play, namely $r_{0 L}$. Although the values of $\sigma(E)$ (considering the 579 nuclei) obtained by minimizing with respect to $r_{0 L}$ and $r_{0 U}$ in the neighborhood of the two possibilities $r_{0 L}=r_{0 U} \approx 0.660 \mathrm{fm}$ and $r_{0 L} \approx 0.490 \mathrm{fm}$ and $r_{0 U}=1.046 \mathrm{fm}$ are similar, the first choice produces a $\Delta B$ plot that looks smoother than the second, and therefore we from now on restrict ourselves to the first choice. We have considered $r_{0 L}=r_{0 U}$ values in the interval between 0.650 and $0.670 \mathrm{fm}$ in steps of $0.002 \mathrm{fm}$ except for the interval bracketing the minimum where a step of $0.001 \mathrm{fm}$ has been considered.
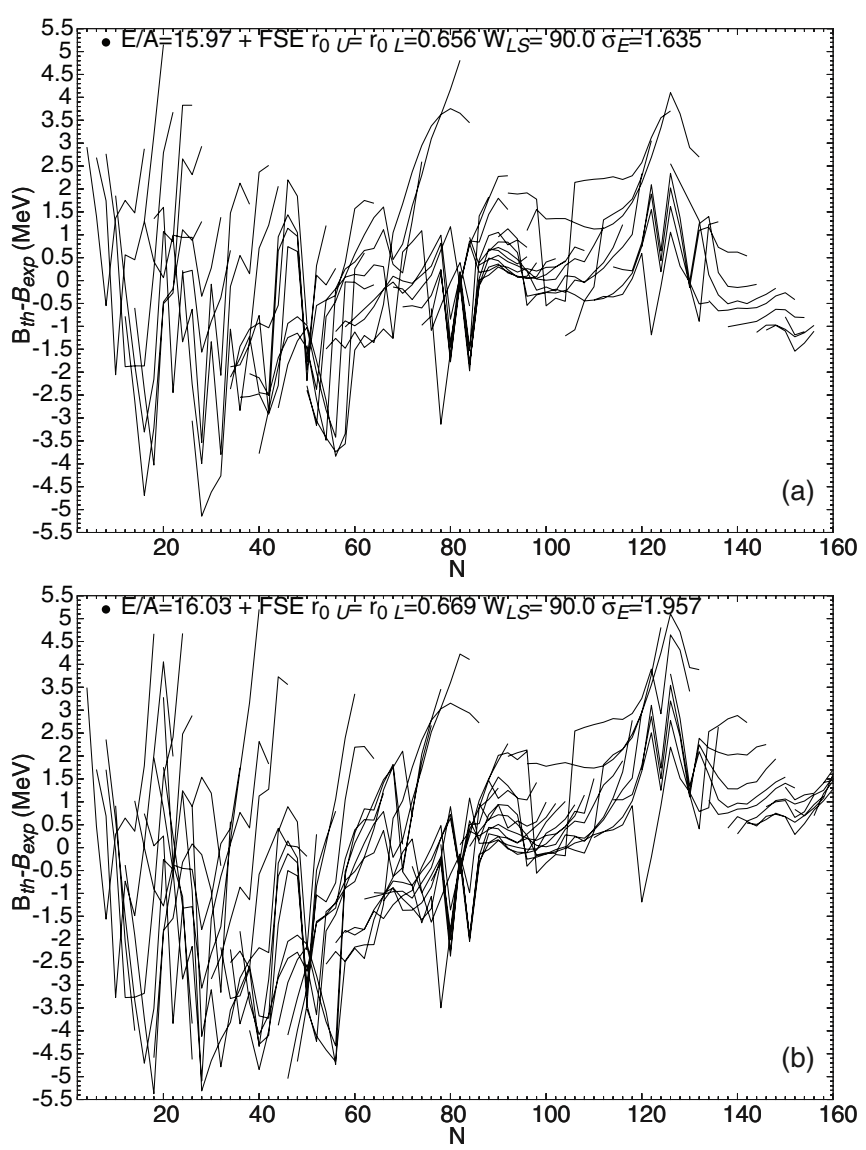

FIG. 2. Binding energy differences $\Delta B=B_{\text {th }}-B_{\exp }$ (in $\mathrm{MeV}$ ) as a function of neutron number $N$ for two choices of $E / A$, namely $E / A=15.97$ in panel (a) and $E / A=16.03$ in panel (b).

After all these considerations, and having carried out a large set of mass table calculations to validate our choice, we arrive to the conclusion that $E / A=15.98 \mathrm{MeV}, W_{L S}=90.5 \mathrm{MeV}$ $\mathrm{fm}^{5}$, and $r_{0 U}=r_{0 L}=0.659 \mathrm{fm}$ is the best choice leading to a $\sigma(E)$ value of $1.58 \mathrm{MeV}$ for the set of 579 nuclei considered in the fit. In the following, as already mentioned, we denote the new set of parameters as the BCPM functional.

Regarding the smallness of the range parameter one should remember that even for a zero range force the surface energy is not zero, owing to the surface spread of the quantum single-particle wave functions. Therefore, it only needs very little extra smearing of the density via a finite range force to get to the experimental value of the surface energy. To quantify this statement, we have calculated the surface energy provided by the BCPM functional using the self-consistent extended Thomas-Fermi approach including $\hbar^{2}$ corrections $\left(\mathrm{ETF}-\hbar^{2}\right)$ [67]. According to this reference, the HF surface energy can be estimated by renormalizing the Weizsäcker term in the semiclassical kinetic energy density. We have obtained the renormalizing factor from a fit to earlier exact $\mathrm{HF}$ calculations (using BCP1 and BCP2 functionals [40]). In this way we estimate the HF surface energy associated with the BCPM functional to be $17.68 \mathrm{MeV}$.

In panel (a) of Fig. 3 we present the $\Delta B$ quantities for the 579 nuclei with measured masses as a function of neutron 

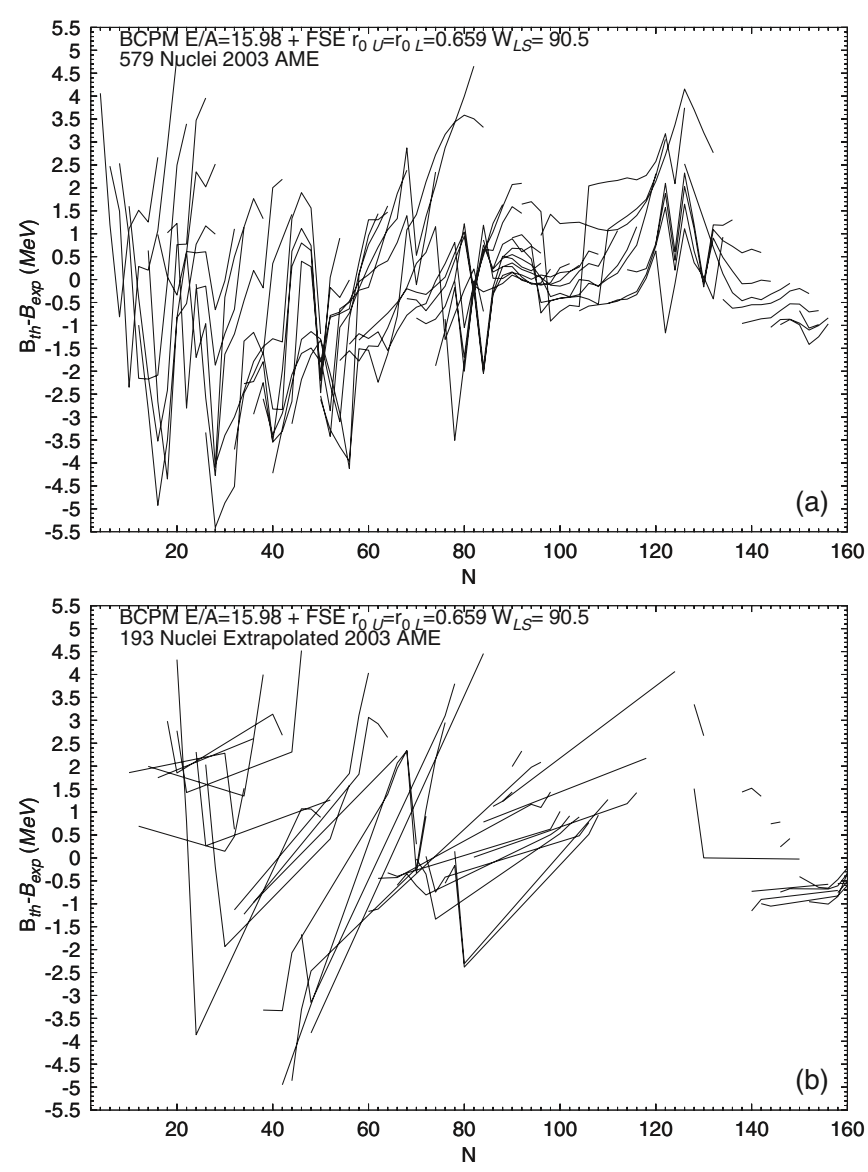

FIG. 3. In panel (a), the binding energy difference $\Delta B=B_{\text {th }}-$ $B_{\exp }($ in $\mathrm{MeV}$ ) is plotted with our optimal set of parameters (BCPM) for the 579 nuclei of Audi's AME 2003 as a function of neutron number $N$. Points corresponding to the same isotope are connected by straight lines. In panel (b), the same quantity is plotted but this time for the extra set of 193 nuclei in Audi's compilation with "experimental" values obtained from extrapolation and/or systematics.

number. The first noticeable conclusion is that, as expected, the agreement with experimental data is much better for heavy nuclei than for light ones. A more quantitative assessment is obtained by looking at $\sigma(E)$ for different sets of nuclei: Taking into account nuclei with $A>40$ (536 nuclei) the $\sigma(E)$ for the energy gets reduced to $1.51 \mathrm{MeV}$ and for the remaining 43 nuclei with $A<40$ it increases up to $2.31 \mathrm{MeV}$. When only nuclei with $A>80$ (452) are considered a $\sigma(E)$ value of $1.34 \mathrm{MeV}$ is obtained. A glance at the plot reveals that those figures are consistent: For heavy nuclei the fluctuations around zero are of small amplitude, whereas for light nuclei the fluctuations lead to discrepancies as high as $5.5 \mathrm{MeV}$ in the upper side or $-5 \mathrm{MeV}$ in the lower one. We also observe strong deviations around magic neutron numbers $N=82$ and $N=126$. In the bottom panel of Fig. 3 we have plotted the $\Delta B$ for those 193 nuclei obtained by extrapolation in the Audi and Wapstra compilation. We observe a very good reproduction of the binding energies for $N \approx 150$ but as $N$ decreases strong deviations from the extrapolations are observed. For the 193 nuclei we obtain $\sigma(E)=2.34 \mathrm{MeV}$, which deviates quite a lot from the nominal value of $1.58 \mathrm{MeV}$. However, the value
TABLE III. Values of $\sigma(E)(\mathrm{MeV})$ for different values of $r_{0 L}$ (fm) in the $r_{0 L}=r_{0 L}$ case. The values of $E / A$ and $W_{L S}$ are fixed at the BCPM values of $15.98 \mathrm{MeV}$ and $90.5 \mathrm{MeV} \mathrm{fm}^{5}$, respectively. Different columns correspond to different choices in the $A$ values included in the evaluation of $\sigma(E)$. For $A \geqslant 40(A \geqslant 80) 536(452)$ nuclei are included.

\begin{tabular}{lccccc}
\hline \hline$r_{0 L}$ & $\sigma(E)$ & $A \geqslant 40$ & $A<40$ & $A \geqslant 80$ & $A<80$ \\
\hline 0.656 & 2.23 & 2.22 & 2.35 & 2.27 & 2.11 \\
0.657 & 1.91 & 1.87 & 2.32 & 1.84 & 2.11 \\
0.658 & 1.68 & 1.62 & 2.31 & 1.52 & 2.16 \\
0.659 & 1.58 & 1.51 & 2.31 & 1.35 & 2.23 \\
0.660 & 1.65 & 1.58 & 2.33 & 1.40 & 2.33 \\
0.661 & 1.85 & 1.82 & 2.35 & 1.64 & 2.45 \\
0.662 & 2.16 & 2.14 & 2.40 & 2.02 & 2.61 \\
\hline \hline
\end{tabular}

for $A>80$ nuclei (146 nuclei) is $1.65 \mathrm{MeV}$, which is much closer to our reference value. For $A<80$ (47 nuclei) we get $\sigma(E)=3.76 \mathrm{MeV}$. The discrepancies can be partly attributed to deficiencies in the extrapolation.

\section{G. Variance of the parameters}

To assess in a more quantitative way the variance of the parameters we have performed three sets of calculations. In the first, the values of $E / A$ and $W_{L S}$ have been kept and different choices for $r_{0 L}=r_{0 L}$ have been made. The results are summarized in Table III, where we observe how a change of $0.003 \mathrm{fm}$ in the $r_{O U}=r_{0 L}$ values (a $0.5 \%$ change) modifies the $\sigma(E)$ values by more than $40 \%$ from 1.58 to $2.16 \mathrm{MeV}$ for $r_{0 U}=r_{0 L}=0.662 \mathrm{fm}\left(2.23 \mathrm{MeV}\right.$ for $\left.r_{0 U}=r_{0 L}=0.656 \mathrm{fm}\right)$. It is also worth mentioning that the $\sigma(E)$ value for $A<$ 40 remains essentially constant as a function of $r_{0 L}=r_{0 U}$, indicating a high degree of randomness in $\Delta B$ for light nuclei that points to a clear deficiency of the mean-field theory to describe such light systems. However, for heavier nuclei with $A>40$ there is a definite parabolic structure with the minimum centered around $r_{0 U}=r_{0 L}=0.659 \mathrm{fm}$. For $A \geqslant 80$ the $\sigma(E)$ value at the minimum gets reduced to $1.35 \mathrm{MeV}$, in agreement with the smaller values of $\Delta B$ observed for heavy nuclei. However, the $A<80 \sigma(E)$ values seem to favor smaller values of $r_{0 U}=r_{0 L}$ but the gain does not seem to be very significant. From the values in the table we can conclude that the use of different values of $r_{0 U}=r_{0 L}$ for different regions of the mass table is only marginally beneficial.

The sensitivity of $\sigma(E)$ to the value of the $r_{0 L}=r_{0 U}$ parameter is a clear indication of the strong connection between this parameter and the contribution of the surface term to the binding energy. To clearly establish this connection we have plotted in Fig. 4 the difference in the HFB energies computed with $r_{0 L}=r_{0 U}=0.659 \mathrm{fm}$ and $r_{0 L}=r_{O U}=0.658$ as a function of $A^{2 / 3}$. The figure shows a clear linear behavior that shows the connection between $r_{0 L}=r_{0 U}$ and the surface energy.

The sensitivity of $\sigma(E)$ to $r_{0 L}=r_{0 U}$ to the level of $0.5 \%$ should not be surprising as it is comparable to the sensitivity of the same quantity to some parameters of Gogny- or Skyrmelike functionals. For instance, the strength parameter $t_{3}$ of 


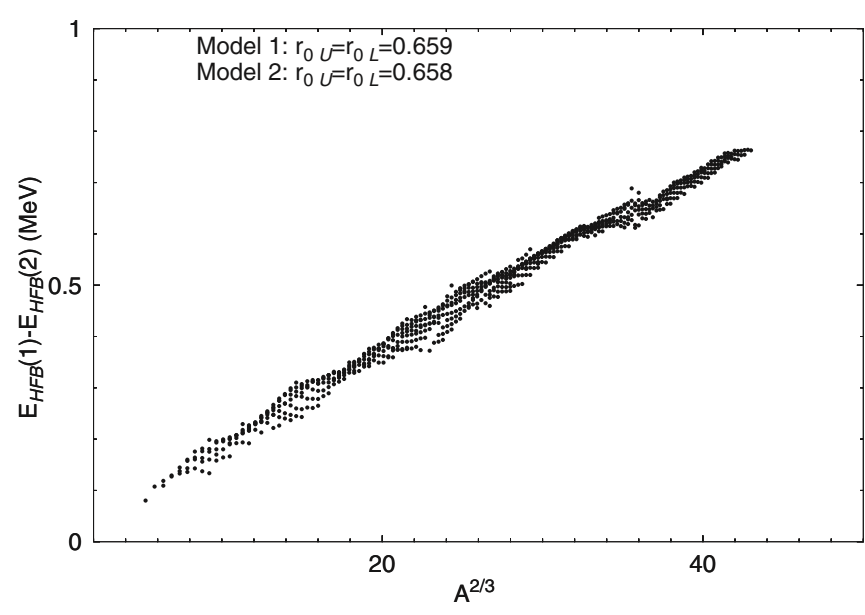

FIG. 4. HFB energy difference computed with $r_{0 L}=r_{0 U}=$ $0.659 \mathrm{fm}$ and $r_{0 L}=r_{0 U}=0.658$ as a function of $A^{2 / 3}$.

the density dependent part of the Gogny D1S EDF produces also strong variations of $\sigma(E)$; a change of $0.1 \%$ in its value increases the $\sigma(E)$ by a factor of 2 (that is a $100 \%$ change). This is not a peculiarity of Gogny D1S because the same sensitivity is observed with Gogny D1M and should be present in any Skyrme with $t_{3}$ values of the order of $10^{3} \mathrm{MeV}$ or higher.

Keeping $E / A$ constant and equal to its optimal value of $15.98 \mathrm{MeV}$ and varying $W_{L S}$ with $r_{0 L}=r_{0 L}$ values determined as to minimize $\sigma(E)$ leads to the results of Table IV. There we observe that changes of the order of $5 \%$ in $W_{L S}$ lead to changes of the order of $6 \%$ in $\sigma(E)$. This result clearly indicates that, as expected, the dependency of the masses on the spin-orbit strength is rather weak.

The second derivatives of the $\sigma(E)$ with respect to the parameters of the fit have been evaluated and their values are given in Table V. By looking at the last row corresponding to the normalized variables we can read off how a $1 \%$ change in the corresponding value of the variables is going to modify the $\sigma(E)$ value at the minimum. For $W_{L S}$ that $1 \%$ change will lead to a change of $0.16 \mathrm{MeV}$ (the smallest), whereas for $r_{0 U}$ it will imply a change of $3.72 \mathrm{MeV}$ (the largest). We conclude that the quality of our fit is more sensitive to $r_{0 U}$ than to $r_{0 L}$. An eigenvalue analysis of the Hessian matrix for $r_{0 U}$ and $r_{0 L}$ shows that there is a nearly zero eigenvalue for those combinations satisfying $r_{0 U}=-0.35 r_{0 L}$. This implies that $r_{0 U}$ and $r_{0 L}$ are not independent variables at least around their optimal values $r_{0 U}=r_{0 L}=0.659$. The other eigenvalue is $4.17 \times 10^{4}$ and corresponds to the combination $r_{0 U}=2.9 r_{0 L}$.

TABLE IV. Values of $\sigma(E)$ (in MeV) for different choices of $W_{L S}$ (in $\mathrm{MeV} \mathrm{fm}^{5}$ ) and at the fixed value $E / A=15.98 \mathrm{MeV}$. For each $W_{L S}$ the values $r_{0 L}=r_{0 L}$ have been fixed to minimize $\sigma(E)$. The values in parentheses following $\sigma(E)$ represent the number of nuclei used in the evaluation of $\sigma(E)$.

\begin{tabular}{lcccc}
\hline \hline$r_{0 L}(\mathrm{fm})$ & $W_{L S}$ & $\sigma(E)$ & $\sigma(E)(A \geqslant 80,452)$ & $\sigma(E)(A<80,127)$ \\
\hline 0.653 & 85.5 & 1.62 & 1.38 & 2.27 \\
0.659 & 90.5 & 1.58 & 1.34 & 2.23 \\
0.665 & 95.5 & 1.67 & 1.44 & 2.32 \\
\hline \hline
\end{tabular}

TABLE V. Values of the second derivative of $\sigma(E)$ (in $\mathrm{MeV}$ ) with respect to the $W_{L S}$ (in $\mathrm{MeV} \mathrm{fm}^{5}$ ), $r_{0 U}$ (in fm), and $r_{0 L}$ (in fm) parameters. The last row is for the values corresponding to variables normalized to the value 1 for the parameters that minimize $\sigma(E)$.

\begin{tabular}{cccccc}
\hline \hline$x=W_{L S}$ & $x=W_{L S}$ & $x=r_{0 U}$ & $x=r_{0 L}$ & $x=r_{0 U}$ \\
$y=W_{L S}$ & $y=r_{0 L}$ & $y=r_{0 U}$ & $y=r_{0 L}$ & $y=r_{0 L}$ \\
\hline$\frac{\partial^{2} \sigma(E)}{\partial x \partial y}$ & 0.195 & -166.812 & $8.58 \times 10^{4}$ & $1.11 \times 10^{4}$ & $2.99 \times 10^{4}$ \\
$\frac{\partial^{2} \sigma(E)}{\partial \bar{x} \partial \bar{y}}$ & $0.16 \times 10^{4}$ & $-0.99 \times 10^{4}$ & $3.72 \times 10^{4}$ & $0.48 \times 10^{4}$ & $1.30 \times 10^{4}$ \\
\hline \hline
\end{tabular}

Finally, to test the sensitivity of our procedure to changes in the degree of the polynomial fitting symmetric matter EOS, we have considered two alternative fits, one with a fourth-order polynomial and another one with a sixth-order one. The incompressibility coefficient $K_{0}$ in symmetric nuclear matter takes the value $238.55 \mathrm{MeV}$ for $n=4$ ( $n$ represents here the polynomial order) and $226.50 \mathrm{MeV}$ in the $n=6$ case. In both cases, the fine tuning of $E / A$ at saturation gives a value of $15.98 \mathrm{MeV}$ as in our final choice of $n=5$ for BCPM. The fit to finite nuclei with the corresponding functionals leads to slight modifications on the value of $r_{0} \equiv r_{0, U}=r_{0, L}$ but the $\sigma(E)$ value at the minimum changes little. For $n=4$ we get $\sigma(E)=1.69 \mathrm{MeV}$ for $r_{0}=0.649 \mathrm{fm}$ and for $n=6$ we get $\sigma(E)=1.64 \mathrm{MeV}$ for $r_{0}=0.654 \mathrm{fm}$. Clearly, the performance of BCPM in reproducing binding energies does not depend in a significant way on the degree of the polynomial fitting symmetric matter.

\section{H. Isotope chain analysis and the role of quadrupole deformation}

We would like to discuss the binding energy differences $\Delta B$ from a different perspective by looking at its behavior with neutron number for each isotopic chain considered. The quantity is plotted in Fig. 5. In each panel, values for the number of protons $Z$ and the number of neutrons $N_{0}$ signaling the origin of the $x$ axis are given. Perpendicular lines in the $\Delta B=0$ horizontal lines indicate the location of magic neutron numbers. From this plot, we conclude that for medium mass and heavy nuclei the $\Delta B$ curves are rather flat as a function of $N$ except for those $Z$ values corresponding to magic numbers $(Z=50$ and 82$)$ plus or minus two. For lighter nuclei the result is less certain but overall we can say that again values of $Z$ of 38,40 (not magic but a subshell closure) and $Z=28$ and 30 have larger values of $\Delta B$ than the other $Z$ values. The impact on the $\sigma(E)$ can be significant. For instance, if nuclei with $Z=48,50,80$, and 82 are not included in the evaluation of $\sigma(E)$ its value goes down to 1.46 for 514 nuclei. Partial $\sigma(E)$ values corresponding to $A>80$ go from 1.34 (452 nuclei) when all possible $Z$ values are considered to 1.09 (387 nuclei) when the $Z$ values indicated above are not considered. It is also observed that for neutron numbers equal to magic ones plus or minus two the binding energy difference $\Delta B$ always shows peaks. The effect is reinforced when both proton and neutron numbers are magic, in such a way that the two nuclei with the largest $\Delta B$ values are ${ }^{208} \mathrm{~Pb}(Z=82)$ and ${ }^{130} \mathrm{Cd}(Z=48)$. Let us finally mention that the peculiarities of this figure are 


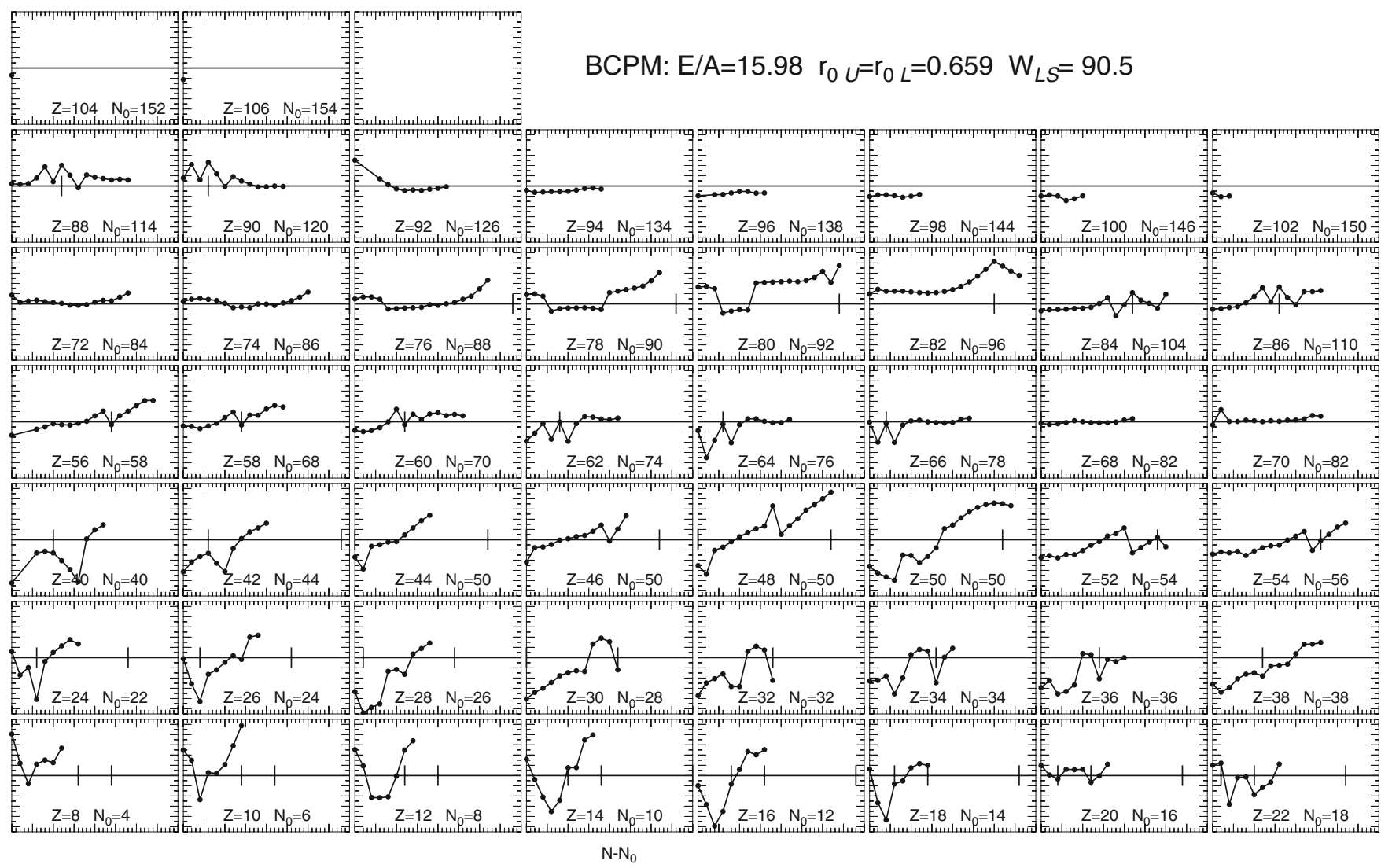

FIG. 5. The binding energy difference $\Delta B=B_{\text {th }}-B_{\text {exp }}(\mathrm{MeV})$ is plotted as a function of the shifted (by $\left.N_{0}\right)$ neutron number $N-N_{0}$ for all the isotopic chains considered. The values of $Z$ and the neutron number shift $N_{0}$ for each chain are given in the corresponding panel. The ordinate $\Delta B$ axis ranges from -5.5 to $5.5 \mathrm{MeV}$ with long ticks every $1 \mathrm{MeV}$. The $N-N_{0}$ axis spans a range of 40 units with long ticks every 5 units and short ones every 1 unit. In every panel, a horizontal line corresponding to $\Delta B=0$ has been plotted to guide the eye. Additional perpendicular lines signaling the position of magic neutron numbers have also been included.

also observed for other forces like Gogny D1S $[61,68]$. The origin of these findings is uncertain but probably has to do with dynamical correlations associated with shape and pairing degrees of freedom.

Another interesting piece of information is the contribution to $\sigma(E)$ according to the deformation of the nuclei. Of the 579 nuclei considered in the evaluation of $\sigma(E)$ there are 365 with a ground-state deformation parameter $\beta_{2}$ greater, in absolute value, than 0.1 (a value that corresponds to a moderately deformed system). The $\sigma(E)$ value for those deformed systems gets reduced to $1.08 \mathrm{MeV}$, whereas the complementary one corresponding to near spherical systems (214 nuclei) grows up to a value of $2.19 \mathrm{MeV}$. This result is in the line of recent claims that well-deformed systems are described better by mean-field models than the spherical ones [69]. The result is also consistent with the previous finding concerning the maximum values of $\Delta B$ for magic or semimagic nuclei as those nuclei tend to have a nearly spherical ground-state shape.

\section{Comparison with other approaches}

In Ref. [70] the idea of using minimax fitting criteria instead of the more traditional rms ones is discussed. The authors considered the Chebyshev norm $\epsilon$, which is based on the maximum absolute value of the residuals $r_{A}=E_{\text {data }}-E_{\text {theory }}$. The object of the fit is to minimize $\epsilon$. The result for SLy4 yields a value of $4.8 \mathrm{MeV}$ with the six critical nuclei ${ }^{20} \mathrm{Mg}$, ${ }^{208} \mathrm{~Pb}$ (both overbound), ${ }^{60} \mathrm{Zn},{ }^{64} \mathrm{Ge}$, and ${ }^{250} \mathrm{Cm}$. We have not carried out a minimax refit of BCPM, but we think the values of the largest residuals are worth being presented. The nuclei with the largest negative $r_{A}$ values are ${ }^{56} \mathrm{Ni}(-5.40 \mathrm{MeV})$, ${ }^{32} \mathrm{~S}(-4.92 \mathrm{MeV})$, and some of their isotopes. On the positive side, we have ${ }^{30} \mathrm{Ne}(4.87),{ }^{130} \mathrm{Cd}(4.65)$, and ${ }^{208} \mathrm{~Pb}(4.15)$. In agreement with the results of Ref. [70], we note that most of the nuclei with large values of $\left|r_{A}\right|$ are magic or semimagic nuclei.

To finish this section we would like to compare our results with those obtained with the different parametrizations of the Gogny force, namely D1S, D1N [71], and D1M [72]. The results for $\sigma(E)$ and the three parametrizations are given in Table VI. For the three parametrizations the values obtained with the raw HFB energies, as well as those including the rotational energy correction (computed according to our procedure), are given. However, the numbers obtained in that way are not very fair in a comparison with BCPM's value because in two cases (D1S and D1N) the binding energies of a few spherical nuclei were used in the fit, and in the other, the binding energy includes quadrupole zero-point energy corrections not taken into account in our calculations. 
TABLE VI. The $\sigma(E)$ values $(\mathrm{MeV})$ for the three parametrizations of the Gogny force D1S [36], D1M [72], and D1N [71] and for different kinds of theoretical calculations. For the row marked as HFB the theoretical binding energy corresponds to the raw mean-field energy, in the one denoted $\mathrm{HFB}+E_{\mathrm{ROT}}$ the rotational energy correction is also included. The last two rows correspond to the same theoretical setup as before but this time a global shift has been applied to the binding energy differences as to minimize the $\sigma(E)$ value. The values of the shift parameter (in $\mathrm{MeV}$ ) for each situation are given in parentheses.

\begin{tabular}{llll}
\hline \hline$\sigma(E)$ & \multicolumn{1}{c}{$\mathrm{D} 1 \mathrm{~S}$} & \multicolumn{1}{c}{$\mathrm{D} 1 \mathrm{M}$} & \multicolumn{1}{c}{$\mathrm{D} 1 \mathrm{~N}$} \\
\hline $\mathrm{HFB}$ & 3.48 & 5.08 & 4.88 \\
$\mathrm{HFB}+E_{\text {ROT }}$ & 2.15 & 2.96 & 2.84 \\
$\mathrm{HFB}+$ Shift & $2.53(2.4)$ & $2.02(4.7)$ & $2.02(4.5)$ \\
$\mathrm{HFB}+E_{\text {ROT }}+$ Shift & $2.14(0.2)$ & $1.47(2.6)$ & $1.45(2.4)$ \\
\hline \hline
\end{tabular}

Therefore, to obtain a number we can compare with BCPM's results we have allowed for a global energy shift of the binding energies with shift values obtained as to minimize the $\sigma(E)$ values (also given in the table). Both D1S and D1N were fitted to the binding energies of just a few spherical nuclei and therefore the impact of the rotational energy correction in the binding energy of deformed nuclei was not included. The effect of adding the rotational correction is noticeable, bringing $\sigma(E)$ down from its original value of 3.48 (4.88) MeV to 2.15 (2.84) MeV for D1S (D1N). The additional phenomenological shift considered reduces the raw $\operatorname{HFB} \sigma(E)$ value substantially in the two cases, but it barely affects the quantity including the rotational energy correction in the D1S case and reduces to a surprisingly good value of $1.45 \mathrm{MeV}$ the $\mathrm{D} 1 \mathrm{~N}$ value. However, D1M is fitted to essentially all the experimentally known binding energies and both a rotational energy correction and a zero-point energy correction associated to quadrupole fluctuations was considered for each nucleus [72]. The raw HFB value given in our table is therefore very large, but the energy shift reduces substantially the $\sigma(E)$ value down to $2 \mathrm{MeV}$. When the rotational energy is included a substantial reduction in $\sigma(E)$ is observed. An additional energy shift (meant in this case to mock up the quadrupole zero-point energy correction, a quantity which is not accessible with our computing resources) brings the $\sigma(E)$ values down to 1.45 MeV. However, this value is still far from the $0.7 \mathrm{MeV}$ given in Ref. [72]. The discrepancy has to be attributed to the slightly different rotational energy corrections and the lack of quadrupole zero-point energies in our calculation. The numbers obtained for D1M and D1N are close to the one of BCPM $(1.58 \mathrm{MeV})$ when the same set of nuclei and beyond-mean-field effects are considered in all the cases. This fact raises the expectation that a BCPM functional fitted with quadrupole zero-point energies (and eventually taking into account the correlation energy of dynamical parity restoration [73] and/or particle number symmetry) could lead to a much smaller value of $\sigma(E)$, well below $1 \mathrm{MeV}$.

Once our model has been established, it is convenient to summarize the procedure used in BCPM and to contrast it with the one of the more standard techniques employed with Skyrme or Gogny functionals. The main difference between the two methods resides in the way the bulk part of the functionals is treated. Within BCPM the latter is adjusted to results of microscopic $G$-matrix calculations (using a polynomial fit). Only a single parameter is fine tuned within $10^{-3}$, which is the energy per particle, $E / A$, at the minimum point. Only the remainder of the functional, which accounts for the inhomogeneity of the system, is fitted with two open parameters to the binding energies of nuclei. So in total there are three parameters which are adjusted to experimental data: $E / A$, a range parameter, and the strength of the spin-orbit term. However, in Skyrme or Gogny functionals, all parameters, bulk and finite size, typically of the order of 12, are, in general, simultaneously adjusted to experiment, sometimes also to a mix of experiment and theoretical data as the neutron EOS, for instance. We think the main advantage of our BCPM functional is this decoupling of parameters: Those implicitly entering the polynomial fit are never used to fine tune the binding energy of finite nuclei exception made of $E / A$.

In Ref. [70] a linear refitting procedure has been proposed to improve the rms deviation of binding-energy differences of various Skyrme-like EDFs. The idea is to start from the parameters of those EDFs and build up gradient and Hessian information that is analyzed using singular value decomposition (SVD) techniques. From the SVD analysis it is concluded that there are three linear combinations of parameters which are relevant: The most important is connected to the volume energy, whereas the next two are linear combinations of surface and symmetry energy coefficients. By performing a refined fit using only those combinations a spectacular improvement of $\sigma(E)$ is obtained for SLy4 going from 3.3 MeV for the 579 even-even nuclei of the 2003 mass table to the value of 1.7 MeV. This result goes along our findings: Once the set of parameters is close to reasonable values only the volume and surface terms (and eventually the symmetry energy) are relevant.

A comparison with Skyrme-like EDFs is also possible, but given the large number of parametrizations available we focus on some recent parametrizations quoted in Ref. [30]. We first mention the UNEDF0 parametrization [74] that includes in the experimental data set to fit not only binding energies but also information on radii and pairing gaps. Special attention is paid also to reproduce nuclear-matter properties at saturation. The fit includes data on both spherical and deformed even-even nuclei and gives an rms for the binding energy of 520 even-even nuclei of $1.45 \mathrm{MeV}$. This is a better value than the one given by BCPM but it is fairly close to it. As a consequence of the poor performance of UNEDF0 in describing fission barrier properties in the actinides (see Ref. [75] for the significance of fission and superdeformation data), a new parametrization denoted UNEDF1 was generated [76]. It includes extra data on fission barrier heights and fission isomer excitation energies of several actinides. The rms for the binding energy of 555 even-even nuclei given by the new parametrization is $\sigma(E)=1.9 \mathrm{MeV}$, which is worse than the $\sigma(E)$ obtained by UNEDF0. In Ref. [77] a survey of Skyrme-like parametrizations was performed. Several observables were included in the fit and several sets of parameters with different nuclear matter properties were considered. This is a completely different strategy from the 
one adopted here, but for the matter of comparison let us quote the rms value of $1.6 \mathrm{MeV}$ for the binding energy of one of the parametrizations obtained, namely SV-min, using 513 experimental masses of even-even nuclei [78]. However, the most recent mass model produced by the Montreal-Brussels collaboration HFB-21 produces a $\sigma(E)$ of $0.58 \mathrm{MeV}$ but for all even- and odd- $A$ nuclei (2149 masses). This figure is obviously much better than the one by BCPM and other approaches discussed above but the number of nuclei considered is larger and also the mass model includes some phenomenological aspects not considered here.

\section{J. Role of the low-density part in the BCPM functional}

One may wonder how significant is the detailed density behavior of the microscopic input below saturation. For instance, the low-density part gives more attraction than most of the more phenomenological mean-field approaches. It is not the purpose of this paper to investigate this question in detail here because it was already discussed in an earlier paper [18]. However, for a first test of the ingredients that make up our functional, we have analyzed the impact of considering instead of the full EOS a parabolic approximation to it. The parabolic approximation is obtained from the series expansion of the microscopic EOS around saturation $E_{\mathrm{Par}}(\rho) \approx E\left(\rho_{0}\right)+$ $k\left(\rho-\rho_{0}\right)^{2}$. It has the obvious disadvantage of reaching a finite value at zero density, which is unphysical. To correct this unwanted behavior another quadratic approximation has also been considered, namely $E_{\text {Quad }}=\epsilon_{1} \rho+\epsilon_{2} \rho^{2}$. It goes to zero at zero density, and the two parameters are adjusted to have the correct minimum at saturation. As a consequence of the

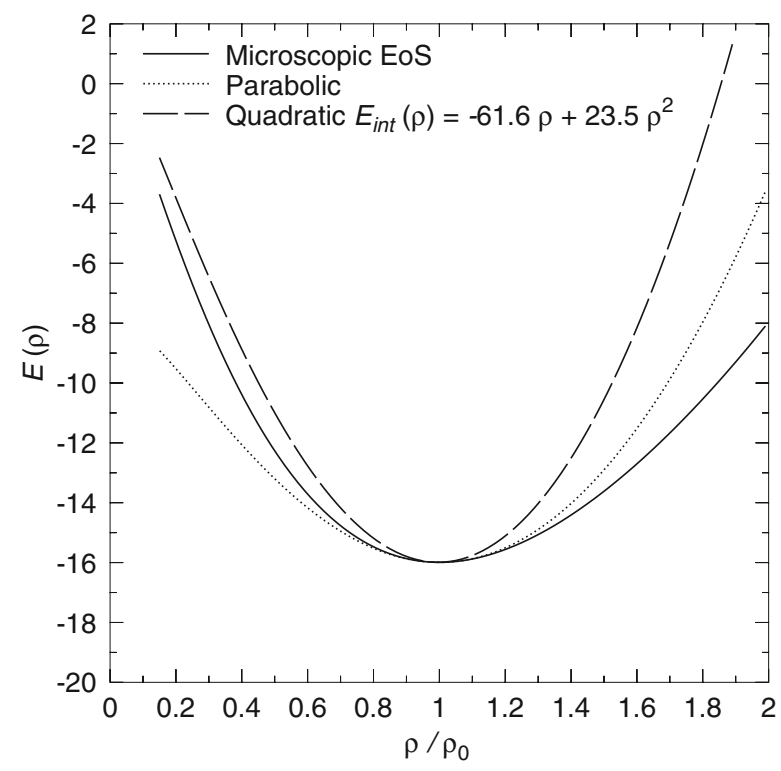

FIG. 6. The different equations of state considered are plotted as a function of the density $\rho$ in units of the saturation density, $\rho_{0}$. The microscopic EOS is the one used to derive the BCPM functional (full line), whereas the parabolic one (dotted line) is the one obtained by a parabolic fit to the microscopic EOS at saturation. Finally, the quadratic EOS is given by the dashed line. In the legend the parameters of the quadratic EOS are shown.

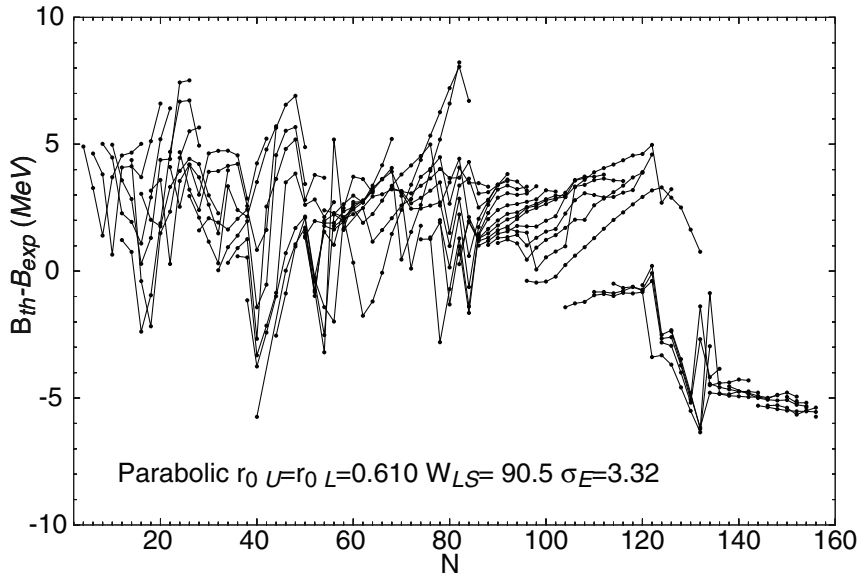

FIG. 7. Binding energy differences $\Delta B=B_{\text {th }}-B_{\text {exp }}(\mathrm{MeV})$ as a function of $N$ obtained for the EDF corresponding to the quadratic approximation $E_{\text {Quad }}=\epsilon_{1} \rho+\epsilon_{2} \rho^{2}$.

limited number of parameters, it is not possible to modify the curvature of $E(\rho)$ around saturation. In Fig. 6 we plot the three EOS considered as a function of density.

The finite nuclei results with the parabolic approximation turn out to be rather poor, probably as a consequence of the behavior at zero density. However, the quadratic approximation results turn out to be quite reasonable in spite of the $\sigma(E)$ value of around $10 \mathrm{MeV}$ obtained without fitting the $r_{0 L}=r_{0 U}$ range parameters. This high value comes essentially from superheavy nuclei (see below). If the range parameters are searched for to minimize the $\sigma(E)$ we obtain a much reduced value of 3.32 (see Fig. 7). The binding energy difference plot still shows the problems with the superheavies mentioned above and we observe that the drop in the binding energy difference for neutron numbers in excess of 130 is completely different from the rest, which looks rather reasonable. The behavior of the superheavies may have to do with the fact that there is an extreme balance of Coulomb, surface, and shell energies and any slight perturbation of the full solution may lead to a strong failure. In conclusion, we can say that a quadratic fit to the EOS may work reasonably well but for optimal numbers, the details of the microscopic density dependence of the EOS below saturation are important.

\section{PERFORMANCE OF THE NEW PARAMETRIZATION FOR OTHER QUANTITIES}

Nuclear binding energies play an important role in the description of atomic nuclei but there are other relevant observables in nuclear dynamics and therefore any functional expected to become "universal" has to perform well also in the description of those quantities. We now discuss a couple of the most relevant ones.

\section{A. Radii}

The nuclear charge radius is a relevant observable connected to many other physical quantities. Experimentally, it can be accurately measured using electron scattering or other complementary techniques. Theoretically, already at the 

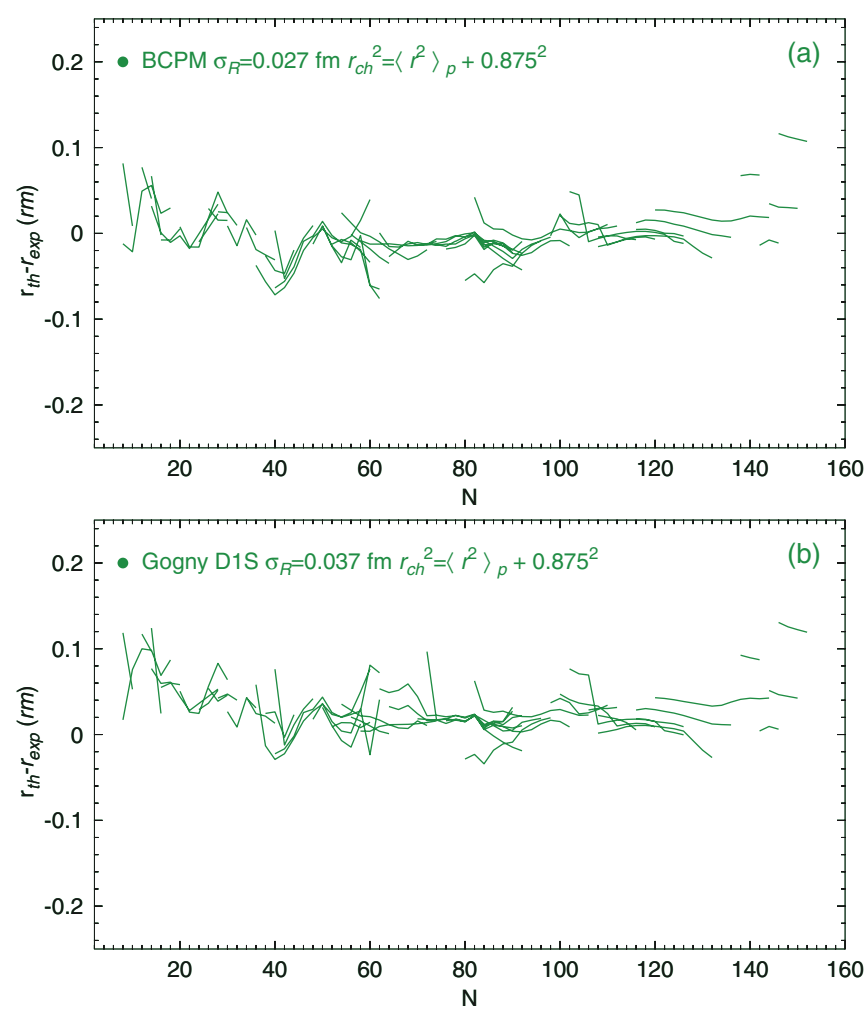

FIG. 8. (Color online) Differences $\Delta r=r_{\text {th }}-r_{\text {exp }}$ between the computed radii and the experimental data taken from Refs. [79-81]. (a) The results for the BCPM functional; (b) the results for the Gogny D1S force. A proton's radius value of $0.875 \mathrm{fm}$ has been considered.

mean-field level it is possible to obtain reasonable values for charge radii if the proton radius is taken into account. The charge radii for all even-even nuclei considered has been computed and compared to the experimental data published in Angeli's compilation [79], except for some $\mathrm{Sr}$ and $\mathrm{Zr}$ isotopes where laser spectroscopy results have been considered instead [80,81]. The theoretical predictions (computed with a proton's radius of $0.875 \mathrm{fm}$ [82]) are confronted to the measured values of 313 even-even nuclei in panel (a) of Fig. 8 . A rms value of $0.027 \mathrm{fm}$ is obtained, which is a quite reasonable number taking into account that the charge radius has not been considered in the fitting protocol. For comparison, the same quantity for the same nuclei computed with the Gogny D1S force [see panel (b) of Fig. 8] is 0.037 and $0.028 \mathrm{fm}$ with the more recent parametrization D1M [72]. We notice that the largest contributions to the BCPM rms value come from the heaviest nuclei considered, where a strong deviation between theory and experiment is observed. Systematic deviations are also observed in nuclei around $N=40, N=60$, and $N=80$, which are regions of the nuclide chart characterized by the phenomenon of shape coexistence. As for the binding energies, the deviations strongly fluctuate in light nuclei with $N<40$. The same pattern is also observed for the Gogny D1S results, suggesting that the origin for the discrepancies is more likely connected with a poor description of the ground state than with deficiencies of the interactions. A comparison of the two plots reveals that a better figure for $\sigma_{R}$ could be achieved in BCPM if an overall displacement would be performed, i.e., the theoretical values systematically underestimate the experimental values. It may be that size fluctuations (RPA correlations) bring the radii to their correct value as suggested in Ref. [83]. This is a task for the future.

\section{B. Quadrupole and octupole deformations}

The quadrupole deformation parameter of the ground state is another relevant parameter associated with low-energy nuclear properties like $2^{+}$excitation energies or $B(E 2)$ transition probabilities. The connection of the intrinsic deformation parameter $\beta_{2}$ with experimental $B(E 2)$ transition probability values is somehow uncertain as it relies on the strong deformation limit of angular momentum projected theories to obtain the rotational formula [84]. For this reason we have preferred to compare BCPM's predictions with the ones of a well reputed, performing, and predictive effective interaction. We have chosen the Gogny force, D1S (results for the most recent published parametrization of the Gogny force, D1M, are very similar). In the top panel of Fig. 9 a histogram is plotted depicting the number of nuclei with ground-state quadrupole deformation parameters $\beta_{2}$ between $\beta_{2}(n)$ and $\beta_{2}(n)+\delta \beta_{2}$. The discrete $\beta_{2}(n)$ values are given by the sequence $\beta_{2}(n)=\delta \beta_{2} n$, with $n=\ldots,-2,-1,0,1,2, \ldots$. The value $\delta \beta_{2}=0.0125$ has been chosen in such a way that each bin represents roughly $5 \%$ of the typical value of 0.25 for the quadrupole deformation parameter. On the bottom panel we plot a histogram with the difference $\beta_{2}(\mathrm{D} 1 \mathrm{~S})-\beta_{2}(\mathrm{BCPM})$.
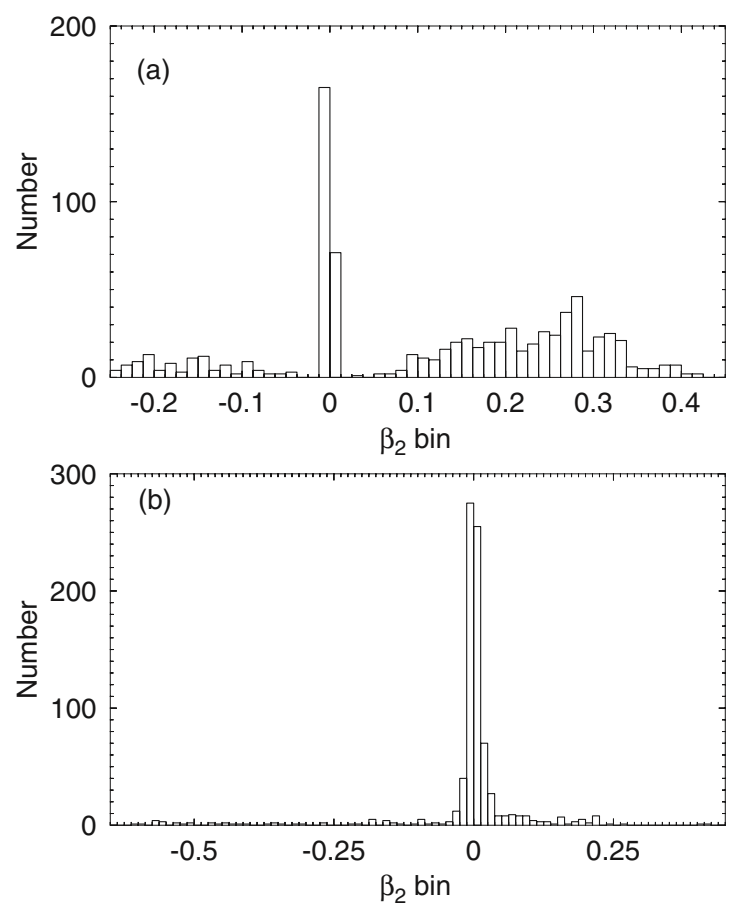

FIG. 9. The number of nuclei with a given quadrupole deformation parameter $\beta_{2}$ in their ground state (in bins 0.0125 units wide) is plotted versus $\beta_{2}$ in panel (a). In panel (b) the number of nuclei with a difference in the ground-state deformation parameter $\beta_{2}$ obtained with BCPM and Gogny D1S is given. The width of the $\beta_{2}$ bins is the same as in panel (a). 
We observe how most of the 818 nuclei considered have a difference of less than 0.0125 in absolute value. A more detailed analysis of the results reveals that most of the discrepancies take place in the region $Z \approx N \approx 40$, which is widely recognized as a region of prolate-oblate shape coexistence and triaxiality.

Octupole deformation is associated with the multipole operator of order three and breaks the parity symmetry. It is not as common as quadrupole deformation in the ground state of atomic nuclei as very specific combinations of orbitals around the Fermi surface have to occur to favor it. Known regions showing octupole deformation are the region around the ${ }^{224} \mathrm{Ra}$ $(Z=88, N=136)$ and the region around ${ }^{144} \mathrm{Ba}(Z=56$ and $N=88)$. We have tested the octupole properties of $\mathrm{BCPM}$ by relaxing the parity symmetry in our self-consistent calculations. In this way, the system can end up in an octupole deformed configuration in its quest for the minimum energy. The results show energy gains of the order of a few hundred $\mathrm{keV}$ and up to $1 \mathrm{MeV}$ in nuclei in the aforementioned regions and both the energy gains and values of the $\beta_{3}$ deformation parameters are compatible with those obtained with the Gogny interaction [73]. Overall, the octupole correlation energies are about a few hundred keV smaller than the corresponding ones obtained with Gogny D1S and D1M ones. Typical examples are the case of ${ }^{224} \mathrm{Ra}$ with octupole correlations energies of 0.63 and $1.31 \mathrm{MeV}$ for BCPM and Gogny D1S, respectively, or the one of ${ }^{144} \mathrm{Ba}$ where the corresponding values are 0.15 and $0.68 \mathrm{MeV}$ for BCPM and Gogny D1S, respectively. For D1S there are five nuclei with mean-field octupole correlation energies larger than $1 \mathrm{MeV}$ and another nine with energies between 1.0 and $0.5 \mathrm{MeV}$. For BCPM the numbers are two with energies above $1 \mathrm{MeV}$ (by just a few $\mathrm{keV}$ ) and nine in the interval 1.0 to $0.5 \mathrm{MeV}$. The situation is similar to the case of BCP1 studied in Ref. [42], with the only difference that in BCP1 the mean-field octupole correlation energy was comparable to the one of Gogny D1S. However, as a consequence of the larger collective masses, the excitation energies predicted by BCP1 were substantially lower than the ones of Gogny D1S and the experiment. It remains to be tested what would be the spectroscopic predictions of BCPM concerning negative parity excited states but the lower mean-field correlation energies go in the right direction.

\section{Fission properties}

Another testing ground for quadrupole and octupole properties of atomic nuclei is the shape evolution from the ground state to spontaneous fission. For this reason we have performed fission barrier calculations for a few selected examples and the results obtained for fission barrier heights and widths as well as mass asymmetry near scission (connected with fragment's mass asymmetry) are quite close to the results obtained with D1S, a functional that was specifically tailored to describe fission properties [36]. A detailed account of these calculations will be presented elsewhere and here we focus on describing the results for a couple of examples: One is the paradigmatic case of ${ }^{240} \mathrm{Pu}$ whose fission properties have been computed with almost any proposed interaction. The other is the superheavy ${ }^{262} \mathrm{Sg}$ where experimental data on spontaneous fission exist. In both cases, the results will be compared with the benchmark calculations carried out with the Gogny EDF. For an early account of fission barrier properties with previous versions of the $\mathrm{BCP}$ functional (BCP1 and $\mathrm{BCP} 2$ ) the reader is referred to Ref. [85].

We have followed the standard procedure that consists in the evaluation of the HFB energy as a function of the mass quadrupole moment $Q_{20}$ with the other multipole moments free to adopt any value to minimize the energy. Along this path to fission, we compute the collective inertias $B\left(Q_{20}\right)$ required to evaluate the spontaneous fission half-life in the WKB approximation (see Ref. [85] for details). The relevant quantities are shown in Figs. 10 and 11 for the nuclei ${ }^{240} \mathrm{Pu}$ and ${ }^{262} \mathrm{Sg}$, respectively. In the bottom panels, the HFB energy is depicted as a function of $Q_{20}$ for both BCPM and Gogny D1S cases. In the two considered nuclei the shape of the energy landscape looks rather similar regardless of the interaction considered. The maximum and minimum are located roughly at the same position and it is only the height of the barriers that

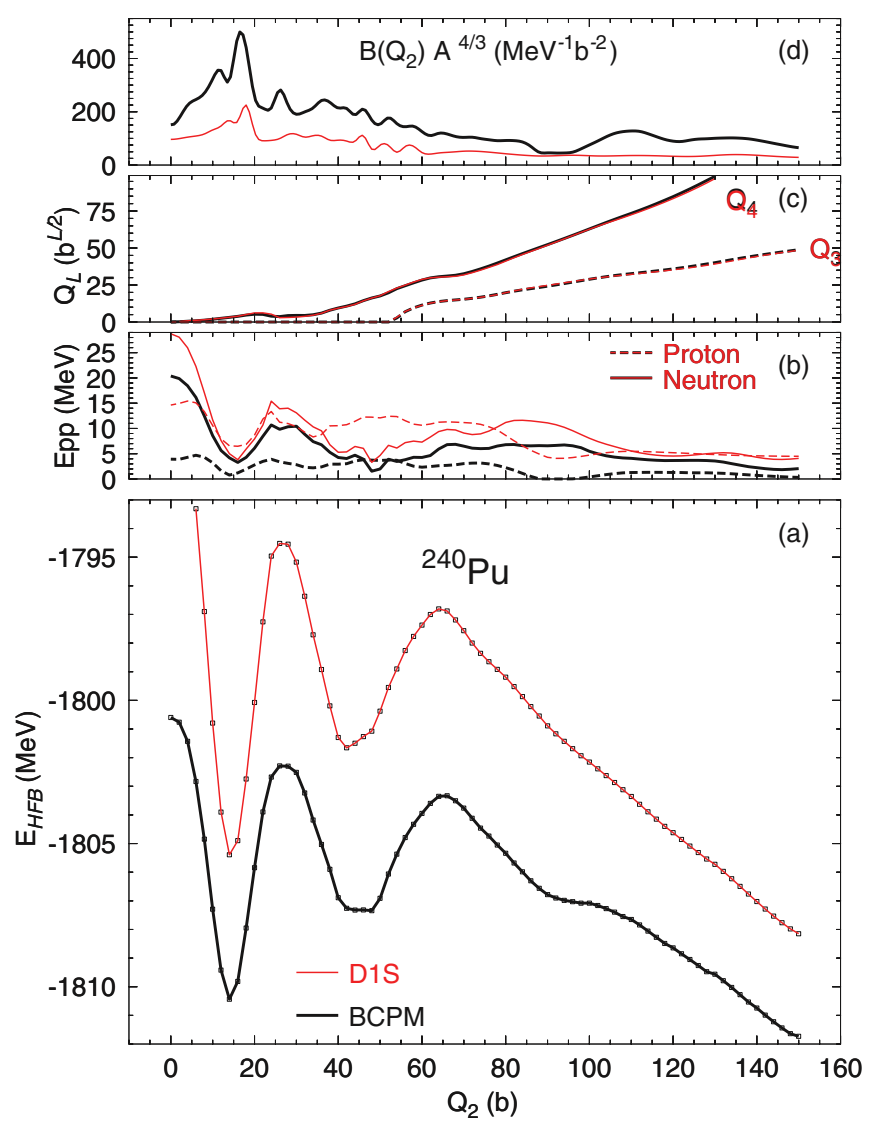

FIG. 10. (Color online) Fission properties of ${ }^{240} \mathrm{Pu}$ obtained with the Gogny D1S interaction (red) and the BCPM functional (thick black). In panel (a) the HFB energy is depicted as a function of the mass quadrupole moment, from the spherical up to a very elongated configuration corresponding to $Q_{2}=150 \mathrm{~b}$. In the other three panels, quantities relevant for the fission half-life are given: in panel (b) the particle-particle correlation energies for protons (dashed lines) and neutrons (full lines); in panel (c) the octupole and hexadecapole moments; and finally in panel (d) the collective mass for quadrupole motion. 


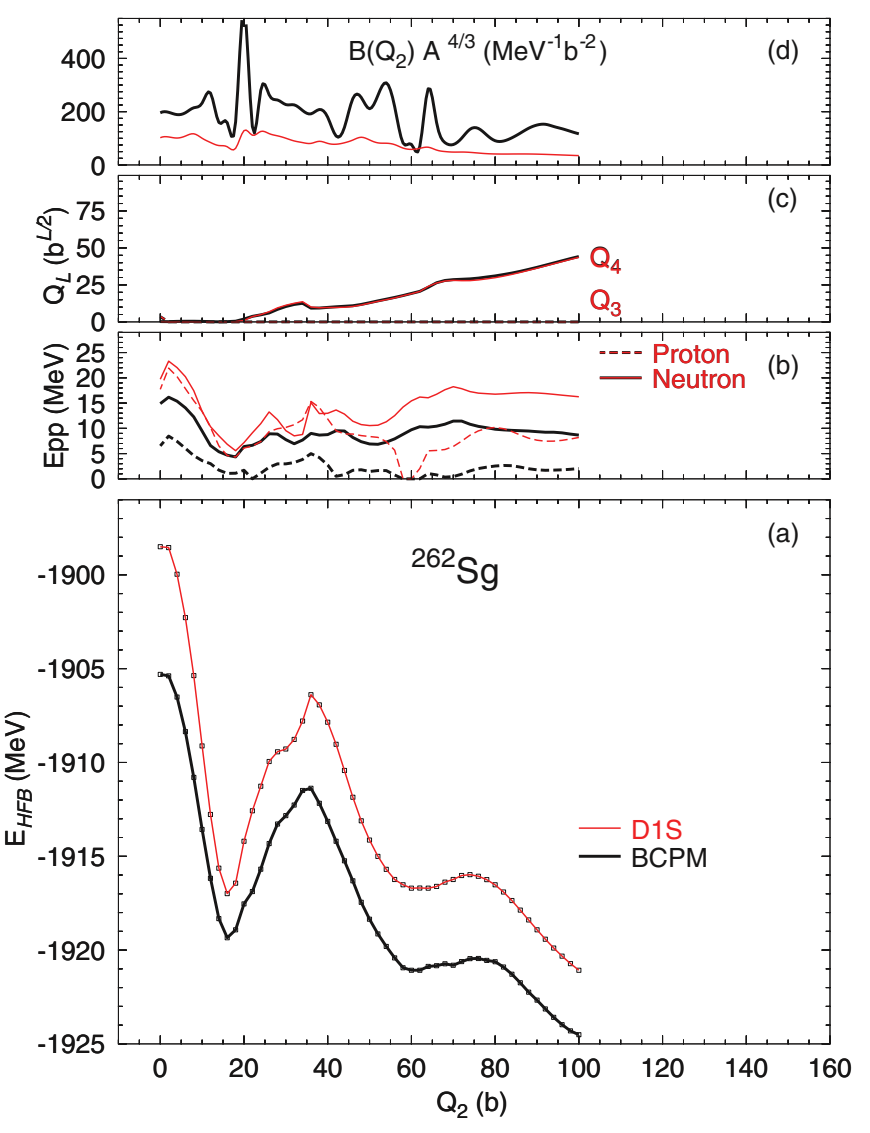

FIG. 11. (Color online) Fission properties of the superheavy nucleus ${ }^{262} \mathrm{Sg}$. See Fig. 10 for the figure caption.

changes with the interaction. Overall, Gogny D1S produces barriers higher than BCPM, in agreement with the larger surface energy coefficient of D1S. The relevance of this characteristic of BCPM depends upon the reduction of the height of the first fission barrier when triaxial shapes are included in the calculation. In the case of the Gogny forces, the reduction can be as large as two or three $\mathrm{MeV}$ but we do not have a hint for BCPM yet. Work is in progress to explore the characteristics of BCPM regarding triaxial shapes. In panel (c) of Figs. 10 and 11, the octupole and hexadecapole moments are depicted as a function of $Q_{20}$ for both interactions. The results for Gogny D1S and BCPM lie one on top of each other and cannot be distinguished in the plot. We observe that in ${ }^{240} \mathrm{Pu}$ octupole deformation develops in its way to fission, pointing to a dominant mass asymmetric fission mode. However, ${ }^{262} \mathrm{Sg}$ remains reflection symmetric along the whole fission path and therefore symmetric fission is expected to be the dominant mode in this nucleus. In panel (b) of both figures the particle-particle pairing energies are given both for protons (dashed) and neutrons (full) for the two interactions. Again, the agreement in the evolution of these quantities with $Q_{20}$ for the two interactions considered is very good but BCPM produces smaller $p p$ pairing energies, suggesting weaker pairing correlations. The collective inertias are quantities very sensitive to the amount of pairing correlations as they strongly depend on the excitation energies of the low-lying two quasiparticle excitations. In panel (d) of Figs. 10 and 11 it is observed that the collective inertias of BCPM are between two and three times larger than the ones of Gogny D1S. The impact of the larger inertias (see Ref. [85] for a thorough discussion of this issue) on the spontaneous fission half lives is to make them longer. This effect is the opposite of having lower barrier heights in BCPM as compared to D1S. Therefore, the final values of the half lives will depend on the specific values of the quantities entering the "collective action." If the spontaneous fission half lives are computed in the WKB approximation we obtain for the ${ }^{240} \mathrm{Pu}$ case the values $t_{1 / 2}(\mathrm{sf})=6.2 \times 10^{18} \mathrm{~s}$ for $\mathrm{D} 1 \mathrm{~S}$ and $t_{1 / 2}(\mathrm{sf})=1.2 \times 10^{28} \mathrm{~s}$ for BCPM, to be compared to the experimental value of $3.5 \times 10^{18} \mathrm{~s}$. For the ${ }^{262} \mathrm{Sg}$ case we obtain the values $t_{1 / 2}(\mathrm{sf})=2.96 \mathrm{~s}$ for D1S and $t_{1 / 2}(\mathrm{sf})=4.2 \mathrm{~s}$ for BCPM, to be compared to the experimental value of $6.9 \mathrm{~ms}$. From the above results we conclude that BCPM tends to yield larger values of $t_{1 / 2}(\mathrm{sf})$ than Gogny D1S and those values can be several orders of magnitude larger than in the Gogny D1S case. This is a direct consequence of the larger collective inertia and therefore a direct consequence of the reduced pairing correlations in BCPM. This results could lead us to the conclusion that BCPM should include stronger pairing correlations if the spontaneous half lives of fission are to be improved. However, we have to be aware of the impact on small changes in the parameters entering the WKB formula. For instance, the ground-state energy usually incorporates some sort of zero-point energy correction (denoted $\epsilon_{0}$ in the literature) that strongly influences the WKB values of $t_{1 / 2}(\mathrm{sf})$. Its value has to be connected to the quantum effects associated with quadrupole motion but its exact meaning is still uncertain. As an example of the sensitivity of $t_{1 / 2}(\mathrm{sf})$ to this parameter, let us mention that if we use a value of $\epsilon_{0}=2.5 \mathrm{MeV}$ instead of the value $\epsilon_{0}=1.5 \mathrm{MeV}$ used originally we will obtain half-lives which are 6 to 12 orders of magnitude smaller. In systematic fission calculations [86] the value of $\epsilon_{0}$ is fine tuned for each isotopic chain considered and therefore absolute values for some choice of $\epsilon_{0}$ as given here should not be considered too seriously for a comparison with experiment. Finally, the agreement between BCPM and Gogny D1S in the values of $Q_{30}$ along the fission path indicates that both interactions will produce, at least in a static framework, the same fission fragment mass distributions.

There are three parameters that characterize in a quantitative way the fission process of ${ }^{240} \mathrm{Pu}$ : One is the height of the first fission barrier $E_{A}$, the second is the excitation energy of the fission isomer with respect to the ground state $E_{I I}$, and finally we have the height of the second barrier $E_{B}$. The accepted experimental values for those parameters are $E_{A}=6.05 \mathrm{MeV}$, $E_{I I}=2.8 \mathrm{MeV}$, and $E_{B}=5.15 \mathrm{MeV}$, whereas the BCPM HFB predictions are $E_{A}=8.15 \mathrm{MeV}, E_{I I}=3.09 \mathrm{MeV}$, and $E_{B}=7.10 \mathrm{MeV}$. We notice a rather good agreement, especially taking into account that no fission data have been considered in the fit. The BCPM value of $E_{A}$ is somewhat too high but this can be attributed to not having considered triaxiality in the calculation, an effect that can lower the energy by a couple of $\mathrm{MeV}$. If the rotational energy correction is considered, the theoretical predictions get reduced to 7.04, 1.69 , and $5.31 \mathrm{MeV}$, respectively. These values are much closer to the experimental value than the HFB ones. The only value that seems to fall too low is $E_{I I}$ but the trend is consistent 
with recent estimations that reduce the experimental value to 2.25 $\mathrm{MeV}$ in ${ }^{240} \mathrm{Pu}$ [87].

\section{EXCITATION PROPERTIES OF THE BCP ENERGY DENSITY FUNCTIONAL}

Although the BCPM EDF is basically built up to deal with some properties of the ground state such as energies and radii, it is, however, interesting to check if this functional can also provide useful information about some excited nuclear states, as for example the isoscalar giant monopole and quadrupole resonances, ISGMR and ISGQR, respectively. Giant resonances can be understood as small-amplitude oscillations of nuclei that are the response to an external field generated by hadronic or electromagnetic probes. A useful theoretical framework for describing these oscillations is the RPA [60] that makes it possible to obtain the particle-hole strength function $S(E)$ which describes the response of the nuclei. However, if the strength is concentrated in a narrow region of the energy spectrum, as it is for the case of the monopole and quadrupole oscillations in medium and heavy stable nuclei, the so-called sum-rule approach [50] is a useful tool that makes it possible to estimate the energy of the giant resonances without performing the full RPA calculations. The sum rules are defined as the moments of the strength function as

$$
m_{k}=\int_{0}^{\infty} E^{k} S(E) E .
$$

The calculation of the sum rules is only easy to handle in few particular cases. Examples are just the ISGMR and ISGQR ones. In these cases the sum rules needed to estimate the average excitation energy of these resonances can be simplified. Of particular interest are the cubic-energy, energy, and inverse-energy weighted sum rules, $m_{3}, m_{1}$, and $m_{-1}$, respectively. Once they are determined one can obtain two estimates of the excitation energy of the ISGMR and ISGQR as

$$
\bar{E}_{3}=\sqrt{\frac{m_{3}}{m_{1}}} \text { and } \quad \bar{E}_{1}=\sqrt{\frac{m_{1}}{m_{-1}}} .
$$

Details about the scaling method and constrained HF calculations, which make it possible to determine the $m_{3}$ and $m_{-1}$ sum rules, respectively, are given in the Appendix. In the following we refer to the average energies provided by the scaling method $\bar{E}_{3}$ in Eq. (A18) and constrained HF calculations $\bar{E}_{1}$ in Eq. (A22) as scaled and constrained energies, respectively.

Experimental information about the excitation energy of isoscalar giant resonances in medium and heavy nuclei is obtained through inelastic scattering of $\alpha$ particles of few hundreds of $\mathrm{MeV}$ measured at extremely forward angles. The multipole decomposition analysis makes it possible to extract from the scattering data the strength associated with different multipolarities, in particular those corresponding to monopole and quadrupole. From these strengths, the position and width of the ISGMR and ISGQR peaks can be determined [88-91].

We analyze first the excitation energies of ISGMR $\left(E_{\mathrm{GMR}}\right)$ of several nuclei for which the experimental values are known [88-91]. We compare these values with the theoretical predictions $\bar{E}_{3}$ and $\bar{E}_{1}$ provided by the BCPM EDF. The relevant information about these excitations energies is collected in Tables VII and VIII. As far as we are including in our study tin and cadmium (neglecting the effects of the small deformation) isotopes and ${ }^{144} \mathrm{Sm}$, for which pairing correlations are essential to describe accurately the ground state, we discuss first the role of pairing in the sum rule approach used to estimate $E_{\mathrm{GMR}}$.

The generalization of RPA to the case of nuclei with open shells when pairing correlations are active is the quasiparticle RPA (QRPA). The so-called dielectric theorem, which justifies constrained calculations within QRPA to obtain the $m_{-1}$ sum rule, has been proven recently by Colò and collaborators in Ref. [92]. Also, Khan et al. have shown the validity of the Thouless theorem for the energy-weighted sum rule in the case

TABLE VII. Theoretical $E_{3}$ and $E_{1}$ estimates of the average excitation energy of the GMR including pairing correlations. The $E_{3}$ estimate of the GQR, also including pairing, is also displayed. The experimental energy of the centroid and the corresponding error for the GMR and GQR are also given.

\begin{tabular}{lcccc}
\hline \hline Nucleus & $E_{3}(M)$ & $E_{1}(M)$ & $E_{3}(Q)$ & $\operatorname{Exp}(\mathrm{M})$ \\
\hline${ }^{90} \mathrm{Zr}$ & 19.06 & 18.32 & 13.34 & $17.81 \pm 0.32$ \\
${ }^{144} \mathrm{Sm}$ & 16.44 & 15.62 & 11.45 & $15.40 \pm 0.30$ \\
${ }^{208} \mathrm{~Pb}$ & 13.84 & 10.16 & $13.96 \pm 0.20$ \\
${ }^{112} \mathrm{Sn}$ & 17.75 & 16.83 & 12.36 & $16.1 \pm 0.1$ \\
${ }^{114} \mathrm{Sn}$ & 16.75 & 12.28 & $15.9 \pm 0.1$ \\
${ }^{116} \mathrm{Sn}$ & 17.64 & 16.66 & 12.21 & $15.8 \pm 0.1$ \\
${ }^{118} \mathrm{Sn}$ & 17.53 & 16.55 & 12.15 & $15.6 \pm 0.1$ \\
${ }^{120} \mathrm{Sn}$ & 17.41 & 16.43 & 12.09 & $15.4 \pm 0.2$ \\
${ }^{122} \mathrm{Sn}$ & 16.32 & 12.04 & $15.0 \pm 0.2$ \\
${ }^{124} \mathrm{Sn}$ & 17.18 & 16.21 & 12.44 & $16.50 \pm 0.2$ \\
${ }^{106} \mathrm{Cd}$ & 17.06 & 16.97 & 12.70 & $16.09 \pm 0.19$ \\
${ }^{110} \mathrm{Cd}$ & 18.09 & 16.83 & 12.38 & $15.72 \pm 0.10$ \\
${ }^{112} \mathrm{Cd}$ & 17.85 & 16.73 & 12.29 & 13.10 \\
${ }^{114} \mathrm{Cd}$ & 17.74 & 16.52 & 12.19 & $13.19 \pm 0.1$ \\
${ }^{116} \mathrm{Cd}$ & 17.59 & & $15.40 \pm 0.12$ \\
\hline \hline
\end{tabular}


TABLE VIII. $E_{3}$ and $E_{1}$ estimates of the average excitation energy of the GMR without including pairing correlations.

\begin{tabular}{lcc}
\hline \hline Nucleus & $E_{3}(M)$ & $E_{1}(M)$ \\
\hline${ }^{90} \mathrm{Zr}$ & 19.07 & 18.36 \\
${ }^{144} \mathrm{Sm}$ & 16.46 & 15.77 \\
${ }^{208} \mathrm{~Pb}$ & 14.49 & 13.84 \\
${ }^{112} \mathrm{Sn}$ & 17.81 & 17.07 \\
${ }^{114} \mathrm{Sn}$ & 17.64 & 17.71 \\
${ }^{116} \mathrm{Sn}$ & 17.56 & 16.66 \\
${ }^{118} \mathrm{Sn}$ & 17.42 & 16.55 \\
${ }^{120} \mathrm{Sn}$ & 17.30 & 16.44 \\
${ }^{122} \mathrm{Sn}$ & 17.19 & 16.33 \\
${ }^{124} \mathrm{Sn}$ & 17.08 & 16.21 \\
${ }^{106} \mathrm{Cd}$ & 18.10 & 17.36 \\
${ }^{110} \mathrm{Cd}$ & 17.86 & 17.11 \\
${ }^{112} \mathrm{Cd}$ & 17.74 & 16.98 \\
${ }^{114} \mathrm{Cd}$ & 17.59 & 16.73 \\
${ }^{116} \mathrm{Cd}$ & 17.44 & 16.52 \\
\hline \hline
\end{tabular}

of self-consistent QRPA based on HFB [93]. As far as a simple generalization of the Thouless theorem allows to obtain the $m_{3}$ sum rule [50], it can be expected that this also will be the case in the QRPA framework. In addition, we have also numerically checked that the virial theorem, i.e., the stability against scale transformations of the wave functions in Eq. (A7), is also fulfilled in the HFB approximation. From this discussion we may conclude that the sum rule approach can also be applied in the QRPA case.

In Table VII we display the theoretical scaled and constrained estimates (including pairing) of $E_{\mathrm{GMR}}$ of the nuclei ${ }^{144} \mathrm{Sm},{ }^{208} \mathrm{~Pb},{ }^{90} \mathrm{Zr},{ }^{112-124} \mathrm{Sn}$, and ${ }^{106,110-116} \mathrm{Cd}$ together with the corresponding experimental values [88-91]. These estimates are given by $\bar{E}_{3}$ and $\bar{E}_{1}$, respectively, defined previously, which, as mentioned, fulfill $\bar{E}_{3}>\bar{E}_{1}$ (see the Appendix). The escape width (A23) lies in a window of 2-3 MeV, pointing out that the monopole strength of the considered nuclei is mainly concentrated around well-defined peaks. The influence of pairing correlations on $E_{\mathrm{GMR}}$ has been discussed in earlier literature (see Refs. [48,94] and references therein). We have repeated the scaled and constrained estimates of the $E_{\mathrm{GMR}}$ switching off pairing and the corresponding results are reported in Table VIII. Comparing Tables VII and VIII we notice that, when pairing correlations are taken into account (Table VII), the $\bar{E}_{1}$ and $\bar{E}_{3}$ values in open-shell nuclei slightly decrease as compared with the values obtained without pairing (Table VIII), which is in agreement with the results of Refs. [48,94]. The effect of pairing correlations is, in general, more important in the constrained calculations than in the scaled ones, especially in half-filled major shell nuclei, as is the case of ${ }^{112} \mathrm{Sn},{ }^{114} \mathrm{Sn},{ }^{116} \mathrm{Sn},{ }^{106} \mathrm{Cd},{ }^{110} \mathrm{Cd},{ }^{112} \mathrm{Cd}$. When the occupancy of the shell increases, as it happens in ${ }^{118} \mathrm{Sn}$, ${ }^{120} \mathrm{Sn},{ }^{122} \mathrm{Sn},{ }^{124} \mathrm{Sn},{ }^{114} \mathrm{Cd}$, and ${ }^{116} \mathrm{Cd}$, the influence of pairing is actually very small, as can be seen in Table VIII.

To compare with the experiment, we use as representative the constrained energy $\bar{E}_{1}$, which estimates the energy of the centroid in a very precise way at least in the case of the nucleus in ${ }^{208} \mathrm{~Pb}[95,96]$ (see also Table IV of Ref. [90] in this respect). From Table VII, we see that the experimental $E_{\mathrm{GMR}}$ in ${ }^{208} \mathrm{~Pb}$ is very well reproduced by $\bar{E}_{1}$ obtained using the BCPM EDF. The experimental excitation energies of ${ }^{90} \mathrm{Zr}$ and ${ }^{144} \mathrm{Sm}$ are slightly overestimated by the theoretical constrained calculation. However, our theoretical calculations are less successful in the case of the cadmium and tin isotopes. The predicted $\bar{E}_{1}$ values are shifted by around $1 \mathrm{MeV}$ up as compared with the experimental results [90,91]. This is a general tendency shown by mean-field models of different nature $[97,98]$. Several attempts to explain this behavior have been proposed in the past such as the density dependence of the incompressibility in neutron rich-matter [99] or different types of pairing interactions [48]. However, no clear explanation has been found to date and the question of why Sn (and Cd) isotopes are so soft is still an open problem. It may be that nonlinear effects coming from particle vibration coupling play a role in such nuclei as $\mathrm{Sn}$ isotopes, because we reproduce the stiff doubly magic nucleus of ${ }^{208} \mathrm{~Pb}$ and not the semimagic ones as, e.g., the $\mathrm{Sn}$ isotopes.

From the previous discussion it seems that the $E_{\mathrm{GMR}}$ estimated with the BCPM functional are somewhat high as compared with the experiment in spite of its relatively low incompressibility modulus $K_{0}=216 \mathrm{MeV}$ (see Table II). The fact that effective mean-field models with different values of $K_{0}$ can give similar values of $E_{\mathrm{GMR}}$ in ${ }^{208} \mathrm{~Pb}$ has actually been a general puzzle in the past (see Refs. $[95,97]$ and references therein). The reason is the proportionality between $E_{\mathrm{GMR}}$ and the square root of $K_{0}$ found by Blaizot [100] for Gogny forces. For example, RMF approaches systematically lead to $E_{\mathrm{GMR}}$ comparable to the ones obtained with nonrelativistic theories like Skyrme or Gogny, in spite of the fact that $K_{0}$ values are appreciably larger in relativistic than in nonrelativistic approaches. Some time ago Colò et al. [95] showed that one can construct Skyrme functionals that give reasonable values for $E_{\mathrm{GMR}}$ but with relatively high values of $K_{0}$, i.e., values which approach the ones of RMF theories. As discussed in Ref. [95], the underlying reason for this fact can be attributed to the different density dependence of the symmetry energy at, and around, saturation. In the same reference [95] it is concluded that within nonrelativistic models there is not a unique relation between the value of $K_{0}$ associated with a given model and the energy and the $E_{\mathrm{GMR}}$ predicted by the same model.

To clarify this latest problem a very recent proposal of Khan, Margueron, and Vidaña [101] may be useful. These authors analyzed a relatively large set of Skyrme, Gogny, and RMF interactions, finding that the corresponding density dependent incompressibilities $K(\rho)$ defined in Eq. (6) show a crossing point at a density of about $75 \%$ of the saturation density. This crossing density corresponds roughly to the average density of a heavy nucleus defined as

$$
\rho_{a v}=\frac{1}{A} \int \rho^{2}(\vec{r}) d \vec{r} .
$$

The authors claim that the excitation energy of finite nuclei is more properly correlated with the slope of incompressibility $K(\rho)$ computed at the crossing density than with the incompressibility modulus computed at saturation density $K_{0}$. This scenario is similar to the one found for the nuclear symmetry 
energy in bulk matter, which also shows a crossing point for a similar value of the density $\left(\simeq 0.11 \mathrm{fm}^{-3}\right)$ [54]. In this case it is also found $[54,56]$ that the slope of the symmetry energy at the crossing density is linearly correlated with the neutron skin of a heavy nucleus, e.g., ${ }^{208} \mathrm{~Pb}$. In general, the existence of a crossing point is actually related to the fact that the parameters of successful mean-field models have been fitted to reproduce properties of finite nuclei whose densities are, on the average, smaller than the saturation density. Therefore, if an observable measured in finite nuclei is related to some property of nuclear matter, the correlation is better described at the average (crossing) density than at the saturation one [101].

Following the suggestion of Ref. [101], we have analyzed the behavior of $E_{\mathrm{GMR}}$ of ${ }^{208} \mathrm{~Pb}$ and ${ }^{90} \mathrm{Zr}$ calculated using different mean-field models as a function of the parameter $M$ defined as

$$
M=\left.3 \rho \frac{d K(\rho)}{d \rho}\right|_{\rho_{a v}},
$$

which is directly related to the slope of the density-dependent incompressibility $K(\rho)$ at the crossing density $\rho_{a v}$. In our study, in addition of the BCPM EDF, we have also considered the D1 and D1S Gogny forces, the SkM* and T6 Skyrme interactions, and the RMF parametrizations NL1, NL3, and NLSH. Using these models the correlation between the scaled and constrained estimates of $E_{\mathrm{GMR}}$ 's and the parameter $M$ are displayed in Fig. 12 for the two aforementioned nuclei. The data of the $E_{\mathrm{GMR}}$ predicted by Gogny forces and RMF parametrizations are taken from Refs. [102] and [103], respectively.

From this figure it can be seen that the $E_{\mathrm{GMR}}$ follows a linear trend with the parameter $M$ (the correlation parameter is $r=0.985$ in all the considered cases) with high accuracy, in agreement with the suggestion of Ref. [101]. Consequently, and as it is claimed in Ref. [101], the density dependence of $K(\rho)$ can be constrained by the $E_{\mathrm{GMR}}$ measured in finite nuclei. The same correlation is also fulfilled by the nucleus ${ }^{90} \mathrm{Zr}$ with $M$ evaluated at the same average density. This fact points out that the dependence on the mass number of the average density is actually rather weak for medium and heavy nuclei [101]. From the lower panel of this figure we also see explicitly that considering globally mean-field models of different nature, the linearity between $E_{\mathrm{GMR}}$ and the square root of the incompressibility modulus at saturation [100] is lost in some cases, in agreement with the conclusion of Ref. [95].

To investigate this aspect in more detail, we plot in the top panel of Fig. 13 the bulk incompressibility of Eq. (6) as a function of the density. In the bottom panel we show, magnified, the relevant region of the top panel. For densities close to $0.72 \rho_{0}$ the density-dependent incompressibility $K(\rho)$ computed with Skyrme and Gogny forces and the RMF takes similar values around $40 \mathrm{MeV}$. However, the BCPM functional predicts relatively larger values of $K(\rho)$ at this average density. The reason for that lies, probably, in the fact that $K(\rho)$ grows more linearly with the density than the other considered models. As far as the slope of $K(\rho)$ has a value consistent with $E_{\mathrm{GMR}}$, as it can be seen in Fig. 12, the value of $K(\rho)$ at $0.72 \rho_{0}$ is larger than the value predicted by the other considered mean-field models. From the analysis of Fig. 13 one
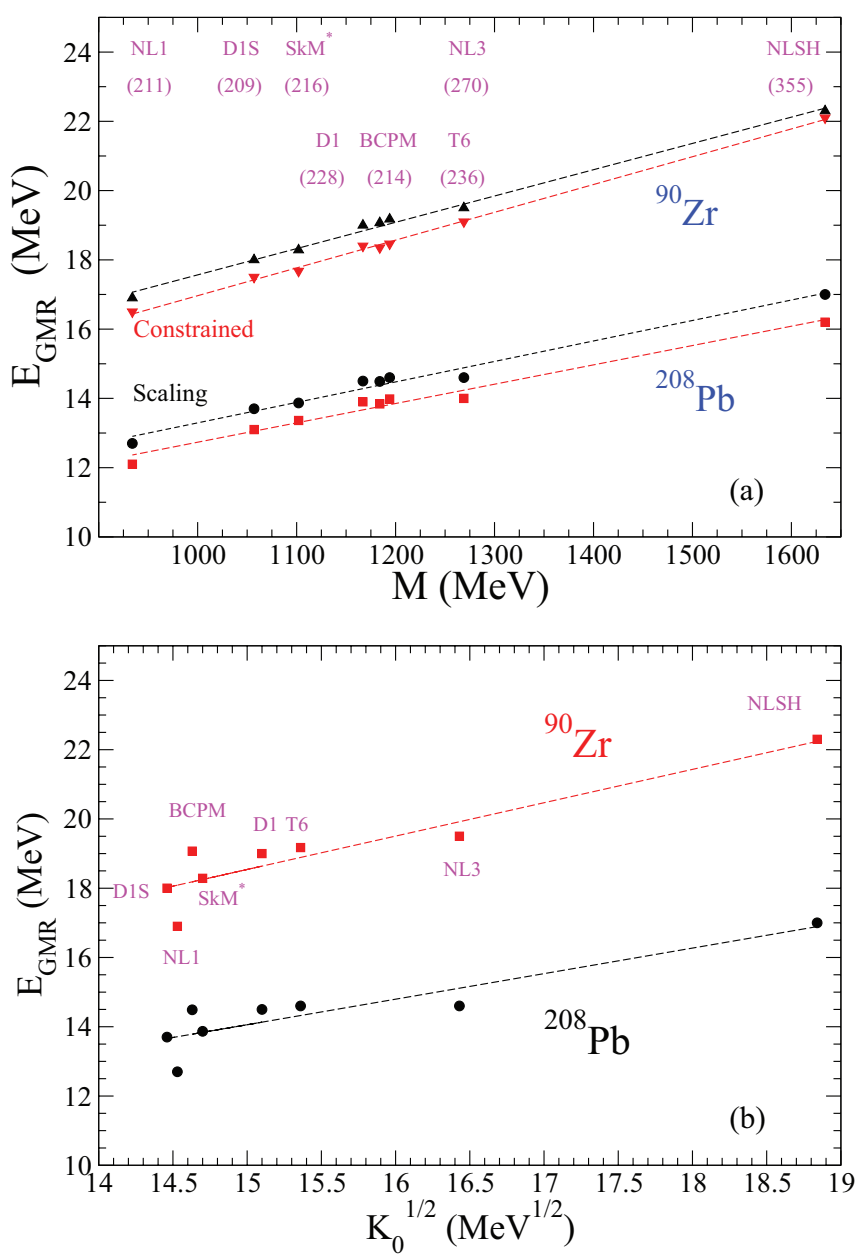

FIG. 12. (Color online) Excitation energies of the GMR of ${ }^{208} \mathrm{~Pb}$ and ${ }^{90} \mathrm{Zr}$ estimated with the scaling method and HF constrained calculations for different mean-field models as a function of the parameter $M$ defined in the text (top). Excitation energies of the GMR of ${ }^{208} \mathrm{~Pb}$ and ${ }^{90} \mathrm{Zr}$ estimated with the scaling approach as a function of the square root of the incompressibility modulus (bottom).

derives two important conclusions. First, the crossing density actually disappears when one considers mean-field models of different nature. What is actually relevant is to compute the slope of $K(\rho)$ at the average density. The same happens in the analysis of the symmetry energy using different mean-field models [56]. Second, the small differences of $K(\rho)$ at the average density may explain why $\sqrt{K_{0}}$ and $E_{\mathrm{GMR}}$ are not always linearly correlated. This happens, e.g., with the NL1 parametrization with $K_{\infty}=211 \mathrm{MeV}$, a value similar to the one of the $\mathrm{SkM}^{*}$ force but predicting $E_{\mathrm{GMR}}$ clearly smaller, or the case of BCPM discussed, previously.

In what concerns the ISGQR, we are well aware of the fact that a theory with $m^{*}=m$, as is the case of the BCPM functional, underestimates the collective quadrupole excitation energy. In Table VII we display the scaled energies of the quadrupole excitation computed with BCPM for several nuclei together with the available experimental values [88-90,104]. Insofar as the focus with a KS-DFT approach is on ground-state properties, we may not worry about the failure in the estimate 

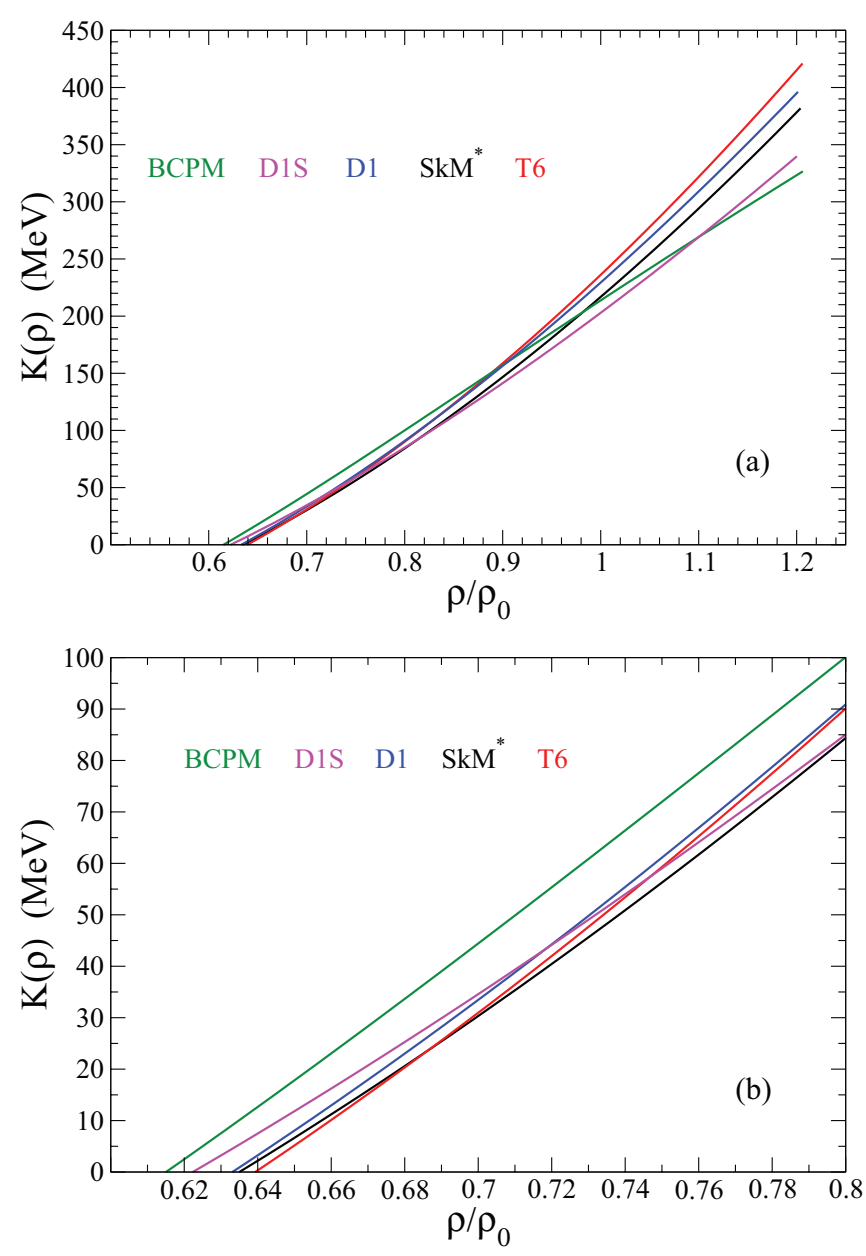

FIG. 13. (Color online) Density-dependent incompressibility calculated using different mean-field models as a function of the density in the ranges $0.5 \leqslant \rho / \rho_{0} \leqslant 1.25$ (top) and $0.6 \leqslant \rho / \rho_{0} \leqslant 0.8$ (bottom).

of the ISGQR. In general, for the description of excitation energies, the KS-DFT should be generalized to include nonlocalities. Attempts in this direction exist in condensed matter. It may be worth looking into that in the future also in the nuclear context.

\section{CONCLUSIONS AND DISCUSSION}

In this work we further elaborated on the previously established KS DFT functional, BCP [1], based on advanced microscopic nuclear and neutron matter calculations. The BCP contained by large five adjustable parameters, three for finite size, one for spin-orbit, and one for bulk. We reduced this number by approximately a factor of two, coming up with a KS-DFT functional containing only three adjustable parameters without deteriorating the excellent results. One of these parameters, the strength of the spin-orbit force, has the usual value, the results being quite insensitive to its variation within a relatively large margin. We would like to call this a rather weak, i.e., almost predetermined parameter (in addition, it may be possible to extract it from the $G$ matrix $[25,105,106])$. The other two parameters, on the contrary, are fixed within extremely tight margins of the order of $10^{-3}$. They concern the bulk energy per particle, $E / A$, on the one hand and the surface energy on the other hand. Indeed, in the polynomial fit to the microscopically determined EOS, a single fine tuning parameter had to be introduced to get a minimal $\mathrm{rms}$ value for nuclear masses as well as a drift free (i.e., scatter around zero) difference of theoretical and experimental binding energies as a function of mass number. This parameter turned out to pin down the binding energy to the value $E / A=15.98 \mathrm{MeV}$. We emphasize that the fourth digit is significant here. For the second parameter, we made the simplifying ansatz to introduce a finite range Gaussian convoluting the two densities in the $\rho^{2}$ terms of nuclear and neutron matter fits, that is, $\rho^{2} \rightarrow$ $\int d^{3} r_{1} d^{3} r_{2} \rho\left(\mathbf{r}_{1}\right) e^{\left(\mathbf{r}_{1}-\mathbf{r}_{2}\right)^{2} / r_{0}^{2}} \rho\left(\mathbf{r}_{2}\right)$. The prefactor is determined from the bulk, so that the only free parameter is the range $r_{0}$ which evidently is responsible for the surface energy. The minimization of the rms of masses (see main text for details) again is determined within the very narrow margin of $10^{-3}$ and turned out to have the value $r_{0}=0.659 \mathrm{fm}$. With this, a rms value for the masses of $1.58 \mathrm{MeV}$ for 579 nuclei and of $0.027 \mathrm{fm}$ for the radii with 313 nuclei is obtained. Those values are as good as, e.g., the ones of the Gogny D1S and D1M forces for the same set of nuclei. This very encouraging result calls for some comments. It is, indeed, quite surprising but very satisfying that the two basic nuclear quantities, energy per particle of the bulk and surface energy essentially suffice to determine a KS-DFT functional with excellent ground-state properties of nuclei. We should point out that the KS-DFT approach is tailored for the ground state and that a time-dependent extension is not evident. We nevertheless tried to determine at least the GMR energies with the sum rule technique and found that the results are in the same ball park as the ones of other Skyrme, relativistic, or Gogny-type approaches. However, the energies of the GQR seem to be underestimated systematically by about $1 \mathrm{MeV}$. This is not so surprising, because a particularity of the KS-DFT approach is that the bare mass is used while it is known that the GQR is sensitive to the value of the effective mass.

Future developments concern eventually the introduction of an effective density-dependent mass $m^{*} \neq m$. Whether this is possible with results as satisfying as here remains to be seen. A further point that needs to be improved is pairing. At the moment, we treat it at a rather elementary level with a zero range force and cutoff. A finite range force of the Gogny type in the pairing channel would certainly be preferable. Promising developments in this direction have recently been published [107]. At the end let us mention that our theory fulfills all the 11 criteria put forward in Ref. [52] except one. Our functional does not agree with the constraint concerning the value of the volume isospin incompressibility coefficient that appears in the leptodermous expansion of the finite nucleus incompressibility [51]. This quantity is defined as

$$
K_{\tau, v}=\left(K_{\mathrm{sym}}-6 L+\frac{K^{\prime}}{K_{0}} L\right) .
$$

The BCPM functional predicts $K_{\tau, v}=-195.2 \mathrm{MeV}$, which is outside of the range proposed in Ref. [52], $-760 \leqslant K_{\tau, v} \leqslant$ $-372 \mathrm{MeV}$. However, two comments are in order. First, the 
extraction of this coefficient from the experimental excitation energies of the GMR in finite nuclei $[52,90,91]$ is not a simple task at all and may give erroneous estimates of the coefficients of the leptodermous expansion of the finite nucleus incompressibility, as has been discussed in the past (see, for example, Refs. [100,108]). Second, there are other estimates of the $K_{\tau, v}$ coefficients available in the literature, as for instance in Ref. [109], that predict $-370 \pm 120 \mathrm{MeV}$, which embraces the prediction of the BCPM functional. In general, the criteria and their limitations in Ref. [52] can certainly be subjected to debate. For example there is no reason to exclude functionals with $m^{*}=m$ as we have demonstrated in this work. The fact that our results comply with the criteria of Ref. [52] is an indication that nuclear density functionals should be based as much as possible on a solid microscopic input. In this respect it is to be noted that, of the five functionals that survived the criteria in Ref. [52], three are also based on a microscopic input.

\section{ACKNOWLEDGMENTS}

We are grateful to P. Danielewicz for useful discussions. We would like to thank N. Schunck for a careful reading of the manuscript and many useful suggestions. The work was supported in part by MICINN Grants No. FPA2009-08958, No. FIS2009-07277, and No. FPA2008-03865-E/IN2P3 and by the Consolider-Ingenio 2010 program CPAN CSD2007-00042 and MULTIDARK CSD2009-00064. X.V. also acknowledges the support from Grants No. FIS2008-01661 (Spain and FEDER) and No. 2009SGR-1289 (Spain). Support by CompStar, a Research Networking Programme of the European Science Foundation, is also acknowledged.

\section{APPENDIX: SUM-RULE APPROACH TO GIANT MONOPOLE AND QUADRUPOLE RESONANCES}

In this Appendix we briefly summarize the basic theoretical aspects of the sum rule approach to obtain moments of the RPA strength functions. For more details, we refer the reader to Refs. [50,60,102,103,110].

In the sum-rule approach it is enough to know few lowenergy weighted moments of $S(E)$ (the so-called sum rules) to estimate the excitation energies of the giant resonances. The sum rules are defined as

$$
m_{k}=\sum_{n} E_{n}^{k}|\langle n|Q| 0\rangle|^{2},
$$

where $Q$ is the operator associated to the excitation, $|0\rangle$ and $|n\rangle$ are the exact ground and excited states, respectively, and $E_{n}$ the excitation energies. If $k$ is an odd integer, the sum rules $m_{k}$ can be computed as the expectation values in the ground state $|0\rangle$ of some commutators which involve the Hamiltonian $H$ and the operator $Q$ (assumed to be a Hermitian and one-body operator) [50], as for example,

$$
m_{1}=\langle 0|[Q,[H, Q]]| 0\rangle
$$

and

$$
m_{3}=\langle 0|[[Q, H],[H,[H, Q]]]| 0\rangle,
$$

from where an average excitation energy can be estimated as

$$
\bar{E}_{3}=\sqrt{\frac{m_{3}}{m_{1}}} .
$$

The full calculation of the sum rules is still a complicated task because the exact ground-state wave function is, in general, unknown. However, if the moments are computed within the $1 \mathrm{p} 1 \mathrm{~h}$ RPA, it is possible to replace the exact ground-state wave function by the uncorrelated $\mathrm{HF}$ one to obtain the sum rules (A2) and (A3) [50]. In spite of all these simplifications, the calculation of the sum rules is only easy to handle in few particular cases, namely the ISGMR and ISGQR ones. In these cases the sum rules needed to estimate the average excitation energy of these resonances can be simplified, as we discuss below.

For the isoscalar monopole and quadrupole oscillations the corresponding excitation operators are taken as

$$
Q_{M}=\sum_{i=1}^{A} r_{i}^{2}, \quad Q_{Q}=\sum_{i=1}^{A}\left(r_{i}^{2}-3 z_{i}^{2}\right)
$$

The underlying effective interaction associated to the BCPM EDF commutes with the operator $Q$ and, therefore, the only contribution to the commutator $[Q,[H, Q]]$ in Eq. (A2) comes from the kinetic energy operator. Consequently $[50,60]$,

$$
m_{1}=\frac{2 \hbar^{2}}{m} A\left\langle r^{2}\right\rangle \quad(L=0), \quad m_{1}=\frac{4 \hbar^{2}}{m} A\left\langle r^{2}\right\rangle \quad(L=2),
$$

where the expectation value of the operator $r^{2}$ is calculated with the HF wave functions.

The direct evaluation of the commutators entering in Eq. (A3) to compute the $m_{3}$ sum rule is a rather cumbersome task that in the case of the monopole and quadrupole oscillations can be avoided with the help the so-called scaling method [50]. We start from the scaled ground-state wave function that in the case of the monopole reads

$$
\phi_{\lambda}^{M}=\lambda^{3 / 2} \phi_{0}(\lambda x, \lambda y, \lambda z),
$$

where $\lambda$ is an arbitrary scaling parameter. In this case the $m_{3}$ sum rule can be expressed by means of the second derivative of the scaled ground-state energy [50], that is,

$$
m_{3}=\frac{1}{2} \frac{\partial^{2}}{\partial \lambda^{2}}\left[\left\langle\Phi_{\lambda}|H| \Phi_{\lambda}\right\rangle\right]_{\lambda=0} .
$$

In the scaling approach the BCPM EDF reads

$$
E(\lambda)=T(\lambda)+E_{\text {int }}^{\infty}(\lambda)+E_{\text {int }}^{\mathrm{FR}}(\lambda)+E_{C}(\lambda)+E^{\text {s.o. }}(\lambda),
$$

where the scaled kinetic, spin-orbit, and Coulomb energies can be found in Ref. [50]. The scaled bulk contribution can be easily obtained from the scaling of the particle density $\left[\rho_{\lambda}(\mathrm{r})=\lambda^{3} \rho(\lambda \mathrm{r})\right]$. The scaling of the finite range contribution has been described in Refs. [102,103]. It is easy to show that the scaling of the Hartee term reduces to a renormalization of the range $\mu$ of the interaction or, in other words, that the scaled relative coordinate is $s_{M, \lambda}=\left|\vec{r}-\vec{r}^{\prime}\right| / \lambda$. In this way, 
the second derivative with respect to the scaling parameter $\lambda$, needed to compute the $m_{3}$ sum rule, finally reads [103]

$$
\left.\frac{d^{2} E_{M}^{H}(\lambda)}{d \lambda^{2}}\right|_{\lambda=1}=\frac{1}{2} \iint d \vec{r} d \overrightarrow{r^{\prime}} \rho(\vec{r}) \rho\left(\vec{r}^{\prime}\right)\left[2 s \frac{d v}{d s}+s^{2} \frac{d^{2} v}{d s^{2}}\right] .
$$

In the BCPM EDF, the form factor $v(s)$ is of Gaussian type with a range $\mu$. In this case the second derivative of the scaled finite range contribution can be recast as [102]

$$
\left.\frac{d^{2} E_{M}^{H}(\lambda)}{d \lambda^{2}}\right|_{\lambda=1}=\mu^{2} \frac{d^{2} E_{M}^{H}(\mu)}{d \mu^{2}}
$$

Finally the $m_{3}$ for the monopole oscillation computed with the BCPM EDF can be written as

$$
\begin{aligned}
m_{3}(L=0)= & \frac{1}{2}\left(\frac{2 \hbar^{2}}{m}\right)^{2}\left[2 T+20 E^{\text {s.o. }}+\left.\frac{d^{2} E^{\infty}(\lambda \rho)}{d \lambda^{2}}\right|_{\lambda=1}\right. \\
& \left.+\mu^{2} \frac{d^{2} E_{M}^{\mathrm{FR}}(\mu)}{d \mu^{2}}\right] .
\end{aligned}
$$

In the case of the quadrupole oscillation the scaling transformation of each single-particle wave function is given by [50]

$$
\rho_{\lambda}(\vec{r})=\rho\left(\lambda x, \lambda y, z^{2} / \lambda^{2}\right) .
$$

Notice that under this transformation the volume element is conserved because of the surface nature of the quadrupole oscillation.

As explained in detail in Ref. [103], a pure Hartree term scales in the quadrupole case as

$$
E_{Q}^{H}(\lambda)=\frac{1}{2} \iint d \vec{r} d \overrightarrow{r^{\prime}} \rho(\vec{r}) \rho\left(\vec{r}^{\prime}\right) v\left(s_{Q, \lambda}\right),
$$

where $s_{Q, \lambda}=\left(s_{x} / \lambda, s_{y} / \lambda, \lambda^{2} s_{z}\right)$. After some algebra the surface contribution to the $m_{3}$ sum rule in the scaling approach for the quadrupole oscillation can be written as (see Ref. [103] for details)

$$
\left.\frac{d^{2} E_{Q}^{H}(\lambda)}{d \lambda^{2}}\right|_{\lambda=1}=\frac{2}{5} \iint d \vec{r} d \vec{r}^{\prime} \rho(\vec{r}) \rho\left(\vec{r}^{\prime}\right)\left[4 s \frac{d v}{d s}+s^{2} \frac{d^{2} v}{d s^{2}}\right]
$$

which in the case of the BCPM functional owing to the Gaussian character of the form factor $v$ can also be written as

$$
\left.\frac{d^{2} E_{Q}^{H}(\lambda)}{d \lambda^{2}}\right|_{\lambda=1}=\frac{4}{5}\left[\mu^{2} \frac{d^{2} E^{H}(\mu)}{d \mu^{2}}-2 \mu \frac{d E_{Q}^{H}(\mu)}{d \mu}\right] .
$$

This is attributable to the fact of the volume conservation in the quadrupole scaling, the bulk interaction part of the BCP EDF, does not contribute to the $m_{3}$ sum rule in this case. The contribution from kinetic and spin-orbit energies can be easily obtained from the scaling transformation of the single-particle wave functions, assuming a spherically symmetric ground state [50]. The Coulomb contribution can also be obtained from Eq. (A14) using the Coulomb form factor $e^{2} / s$ [103]. Collecting all these contributions, the $m_{3}$ sum rule for the quadrupole oscillation can finally be written as

$$
\begin{aligned}
m_{3}(L=2)= & 4\left(\frac{2 \hbar^{2}}{m}\right)^{2}\left\{T+\frac{1}{4} E^{\text {s.o. }}-\frac{1}{5} E_{C}\right. \\
& \left.+\frac{1}{10}\left[\mu^{2} \frac{d^{2} E_{Q}^{H}(\mu)}{d \mu^{2}}-2 \mu \frac{d E_{Q}^{H}(\mu)}{d \mu}\right]\right\} .
\end{aligned}
$$

Once the $m_{3}$ sum rule has been obtained through the scaling method, an estimate of the excitation energy of the ISGMR and ISGQR can be calculated with the help of Eqs. (A4) and (A6):

$$
E_{M}^{S}=\sqrt{\frac{m_{3}}{m_{1}}} .
$$

Let us now consider a nucleus, described by a Hamiltonian $H$, under the action of a weak one-body field $\eta Q$. Assuming $\eta$ sufficiently small, so that the perturbation theory holds, the variation of the expectation value of $Q$ and $H$ are directly related to the $m_{-1}$ moment [50],

$$
m_{-1}=\sum_{n} \frac{|\langle n|Q| 0\rangle|^{2}}{E_{n}}=-\frac{1}{2}\left[\frac{\partial\langle Q\rangle}{\partial \eta}\right]_{\eta=0}=\frac{1}{2}\left[\frac{\partial^{2}\langle H\rangle}{\partial \eta^{2}}\right]_{\eta=0} .
$$

Therefore, at RPA level the average energy of the giant resonances can also be estimated performing constrained HF calculations, i.e., looking for the HF solutions of the constrained Hamiltonian,

$$
H(\eta)=H-\eta Q,
$$

where $Q$ is the collective monopole (quadrupole) operator defined previously [see Eq. (A5)]. From the constrained HF wave-function $\Phi(\eta)$ solution of (A20), the RPA $m_{-1}$ moment can be written as

$$
\begin{aligned}
m_{-1} & =-\frac{1}{2}\left[\frac{\partial}{\partial \eta}\langle\Phi(\eta)|Q| \Phi(\eta)\rangle\right]_{\eta=0} \\
& =\frac{1}{2}\left[\frac{\partial^{2}}{\partial \eta^{2}}\langle\Phi(\eta)|H| \Phi(\eta)\rangle\right]_{\eta=0},
\end{aligned}
$$

from where another estimate of the average energy of the isoscalar giant resonances is given by

$$
\bar{E}_{1}=\sqrt{\frac{m_{1}}{m_{-1}}} .
$$

Owing to the fact that the RPA moments fulfill $\sqrt{m_{3} / m_{1}} \geqslant$ $m_{1} / m_{0} \geqslant \sqrt{m_{1} / m_{-1}}$ [50], the average energies $\bar{E}_{3}$ and $\bar{E}_{1}$ values are an upper and lower bound of the mean energy of the resonance and their difference is related with the variance of the strength function (resonance width)

$$
\sigma=\frac{1}{2} \sqrt{\bar{E}_{3}^{2}-\bar{E}_{1}^{2}} .
$$


[1] M. Baldo, P. Schuck, and X. Viñas, Phys. Lett. B 663, 390 (2008).

[2] W. Kohn and L. J. Sham, Phys. Rev. 140, A1133 (1965).

[3] P. Hohenberg and W. Kohn, Phys. Rev. 136, B864 (1964).

[4] R. O. Jones and O. Gunnarsson, Rev. Mod. Phys. 61, 689 (1989).

[5] H. Eschrig, The Fundamentals of Density Functional Theory (B. G. Teubner, Stuttgart, 1996).

[6] J. P. Perdew and K. Schmidt, Density Functional Theory and Its Applications to Material, edited by V. Van Doren et al. (American Institute of Physics, Melville, NY, 2001), CP577.

[7] A. E. Mattsson, Science 298, 759 (2002).

[8] Jianmin Tao, J. P. Perdew, V. N. Staroverov, and G. E. Scuseria, Phys. Rev. Lett. 91, 146401 (2003).

[9] Jianmin Tao and J. P. Perdew, Phys. Rev. Lett. 95, 196403 (2005).

[10] J. Messud, M. Bender, and E. Suraud, Phys. Rev. C 80, 054314 (2009)

[11] J. Engel, Phys. Rev. C 75, 014306 (2007).

[12] N. Barnea, Phys. Rev. C 76, 067302 (2007).

[13] M. Baldo, I. Bombaci, and G. F. Burgio, Astron. Astrophys. 328, 274 (1997).

[14] M. Baldo, A. Fiasconaro, H. Q. Song, G. Giansiracusa, and U. Lombardo, Phys. Rev. C 65, 017303 (2001).

[15] M. Baldo, G. Giansiracusa, U. Lombardo, and H. Q. Song, Phys. Lett. B 473, 1 (2000).

[16] X. R. Zhou, G. F. Burgio, U. Lombardo, H.-J. Schulze, and W. Zuo, Phys. Rev. C 69, 018801 (2004).

[17] Z. H. Li, U. Lombardo, H.-J. Schulze, W. Zuo, L. W. Chen, and H. R. Ma, Phys. Rev. C 74, 047304 (2006).

[18] M. Baldo, C. Maieron, P. Schuck, and X. Viñas, Nucl. Phys. A 736, 241 (2004).

[19] M. Baldo and C. Maieron, J. Phys. G: Nucl. Part. Phys. 34, R243 (2007).

[20] M. Baldo and G. F. Burgio, Rep. Prog. Phys. 75, 026301 (2012).

[21] G. Taranto, M. Baldo, and G. F. Burgio, Phys. Rev. C 87, 045803 (2013).

[22] S. A. Fayans, JETP Lett. 68, 169 (1998).

[23] A. W. Steiner, M. Prakash, J. M. Lattimer, and P. J. Ellis, Phys. Rep. 411, 325 (2005).

[24] B. Friedman and V. R. Pandharipande, Nucl. Phys. A 361, 502 (1981); R. B. Wiringa, V. Fiks, and A. Fabrocini, Phys. Rev. C 38, 1010 (1988).

[25] J. W. Holt, N. Kaiser, and W. Weise, Phys. Rev. C 81, 024002 (2010).

[26] J. W. Holt, N. Kaiser, and W. Weise, Eur. Phys. J. A 47, 128 (2011).

[27] N. Kaiser, Eur. Phys. J. A 48, 36 (2012).

[28] B. Gebremariam, T. Duguet, and S. K. Bogner, Phys. Rev. C 82, 014305 (2010).

[29] M. Stoitsov, M. Kortelainen, S. K. Bogner, T. Duguet, R. J. Furnstahl, B. Gebremariam, and N. Schunck, Phys. Rev. C 82, 054307 (2010).

[30] J. Erler, N. Birge, M. Kortelainen, W. Nazarewicz, E. Olsen, A. Perhac, and M. Stoitsov, Nature (London) 486, 509 (2012).

[31] M. Baldo, L. Robledo, P. Schuck, and X. Viñas, J. Phys. G: Nucl. Part. Phys. 37, 064015 (2010)

[32] J. Engel, Lecture Notes in Physics, edited by C. Fiolhais, F. Nogueira, and M. Marques, Vol. 620 (Springer-Verlag, Berlin, 2003), p. 56.
[33] E. Chabanat, P. Bonche, P. Haensel, J. Meyer, and R. Schaeffer, Nucl. Phys. A 635, 231 (1998); 643, 441 (1998).

[34] S. Goriely, N. Chamel, and J. M. Pearson, Phys. Rev. Lett. 102, 152503 (2009).

[35] J. Dechargé and D. Gogny, Phys. Rev. C 21, 1568 (1980).

[36] J. F. Berger, M. Girod, and D. Gogny, Nucl. Phys. A 428, 23C (1984).

[37] L. N. Oliveira, E. K. U. Gross, and W. Khon, Phys. Rev. Lett. 60, 2430 (1988).

[38] E. Garrido, P. Sarriguren, E. Moya de Guerra, and P. Schuck, Phys. Rev. C 60, 064312 (1999).

[39] G. A. Lalazissis, J. Konig, and P. Ring, Phys. Rev. C 55, 540 (1997).

[40] L. M. Robledo, M. Baldo, P. Schuck, and X. Viñas, Phys. Rev. C 77, 051301(R) (2008).

[41] X. Viñas, L. M. Robledo, P. Schuck, and M. Baldo, Int. J. Mod. Phys. E 18, 935 (2009).

[42] L. M. Robledo, M. Baldo, P. Schuck, and X. Viñas, Phys. Rev. C 81, 034315 (2010).

[43] J. L. Egido and L. M. Robledo, Lect. Notes Phys. 641, 269 (2004).

[44] J. Margueron, E. van Dalen, and C. Fuchs, Phys. Rev. C 76, 034309 (2007).

[45] A. Akmal, V. R. Pandharipande, and D. G. Ravenhall, Phys. Rev. C 58, 1804 (1998).

[46] L. M. Robledo, Int. J. Mod. Phys. E 16, 337 (2007).

[47] L. M. Robledo, J. Phys. G 37, 064020 (2010).

[48] E. Khan, J. Margueron, G. Colò, K. Hagino, and H. Sagawa, Phys. Rev. C 82, 024322 (2010).

[49] W. D. Myers and W. J. Swiatecki, Ann. Phys. (NY) 55, 395 (1969).

[50] O. Bohigas, A. M. Lane, and J. Martorell, Phys. Rep. 51, 267 (1979).

[51] J. P. Blaizot, Phys. Rep. 64, 171 (1980).

[52] M. Dutra, O. Lourenço, J. S. Sá Martins, A. Delfino, J. R. Stone, and P. D. Stevenson, Phys. Rev. C 85, 035201 (2012).

[53] M. B. Tsang et al., Phys. Rev. C 86, 015803 (2012).

[54] B. A. Brown, Phys. Rev. Lett. 85, 5296 (2000).

[55] M. Warda, X. Viñas, X. Roca-Maza, and M. Centelles, Phys. Rev. C 80, 024316 (2009).

[56] M. Centelles, X. Roca-Maza, X. Viñas, and M. Warda, Phys. Rev. Lett. 102, 122502 (2009).

[57] R. C. Nayak, J. M. Pearson, M. Farine, P. Gleissl, and M. Brack, Nucl. Phys. A 516, 62 (1990).

[58] T. V. Chossy and W. Stocker, Phys. Rev. C 56, 2518 (1997).

[59] G. Audi and A. H. Wapstra, Nucl. Phys. A 729, 337 (2003).

[60] P. Ring and P. Schuck, The Nuclear Many Body Problem (Springer, Berlin, 1980).

[61] S. Hilaire and M. Girod, Eur. Phys. J. A 33, 237 (2007).

[62] L. M. Robledo and G. F. Bertsch, Phys. Rev. C 84, 014312 (2011).

[63] C. Titin-Schnaider and Ph. Quentin, Phys. Lett. B 49, 397 (1974).

[64] M. Anguiano, J. L. Egido, and L. M. Robledo, Nucl. Phys. A 683, 227 (2001).

[65] M. N. Butler, D. W. L. Sprung, and J. Martorell, Nucl. Phys. A 422, 157 (1984).

[66] V. B. Soubbotin, V. I. Tselyaev, and X. Viñas, Phys. Rev. C 67, 014324 (2003).

[67] M. Centelles, M. Del Estal, and X. Viñas, Nucl. Phys. A 635, 193 (1998). 
[68] Gogny D1S web mass table, http://www-phynu.cea.fr/HFBGogny.htm.

[69] T. Niksic, D. Vretenar, and P. Ring, Phys. Rev. C 78, 034318 (2008).

[70] G. F. Bertsch, B. Sabbey, and M. Uusnäkki, Phys. Rev. C 71, 054311 (2005).

[71] F. Chappert, M. Girod, and S. Hilaire, Phys. Lett. B 668, 420 (2008).

[72] S. Goriely, S. Hilaire, M. Girod, and S. Perú, Phys. Rev. Lett. 102, 242501 (2009).

[73] L. M. Robledo and G. F. Bertsch, Phys. Rev. C 84, 054302 (2011).

[74] M. Kortelainen, T. Lesinski, J. Moré, W. Nazarewicz, J. Sarich, N. Schunck, M. V. Stoitsov, and S. Wild, Phys. Rev. C 82, 024313 (2010).

[75] N. Nikolov, N. Schunck, W. Nazarewicz, M. Bender, and J. Pei, Phys. Rev. C 83, 034305 (2011).

[76] M. Kortelainen, J. McDonnell, W. Nazarewicz, P.-G. Reinhard, J. Sarich, N. Schunck, M. V. Stoitsov, and S. M. Wild, Phys. Rev. C 85, 024304 (2012).

[77] P. Klüpfel, P.-G. Reinhard, T. J. Bürvenich, and J. A. Maruhn, Phys. Rev. C 79, 034310 (2009).

[78] P.-G. Reinhard and B. K. Agrawal, Int. J. Mod. Phys. E 20, 1379 (2011).

[79] I. Angeli, At. Data Nucl. Data Tables 87, 185 (2004).

[80] F. Buchinger, E. B. Ramsay, E. Arnold, W. Neu, R. Neugart, K. Wendt, R. E. Silverans, P. Lievens, L. Vermeeren, D. Berdichevsky, R. Fleming, D. W. L. Sprung, and G. Ulm, Phys. Rev. C 41, 2883 (1990).

[81] P. Campbell et al., Phys. Rev. Lett. 89, 082501 (2002).

[82] C. Amsler et al. (Particle Data Group), Phys. Lett. B 667, 1 (2008).

[83] M. Dupuis, S. Karataglidis, E. Bauge, J. P. Delaroche, and D. Gogny, Phys. Rev. C 73, 014605 (2006).

[84] H. J. Mang, Phys. Rep. 18, 325 (1975).

[85] L. M. Robledo, M. Baldo, P. Schuck, and X. Viñas, J. Phys.: Conf. Ser. 321, 012015 (2011).

[86] M. Warda, J. L. Egido, L. M. Robledo, and K. Pomorski, Phys. Rev. C 66, 014310 (2002).

[87] M. Hunyadi et al., Phys. Lett. B 505, 27 (2001).

[88] D. H. Youngblood, Y.-W. Lui, H. L. Clark, B. John, Y. Tokimoto, and X. Chen, Phys. Rev. C 69, 034315 (2004).
[89] D. H. Youngblood, Y.-W. Lui, B. John, Y. Tokimoto, H. L. Clark, and X. Chen, Phys. Rev. C 69, 054312 (2004).

[90] T. Li et al., Phys. Rev. Lett. 99, 162503 (2007); Phys. Rev. C 81, 034309 (2010).

[91] D. Patel et al., Phys. Lett. B 718, 447 (2012).

[92] L. Capelli, G. Coló, and Jun Li, Phys. Rev. C 79, 054329 (2009).

[93] E. Khan, N. Sandulescu, M. Grasso, and N. V. Giai, Phys. Rev. C 66, 024309 (2002).

[94] V. Tselyaev, J. Speth, S. Krewald, E. Litvinova, S. Kamerdzhiev, N. Lyutorovich, A. Avdeenkov, and F. Grümmer, Phys. Rev. C 79, 034309 (2009).

[95] G. Colò, N. V. Giai, J. Meyer, K. Bennaceur, and P. Bonche, Phys. Rev. C 70, 024307 (2004).

[96] E. Khan, Phys. Rev. C 80, 011307 (2009).

[97] G. Colò and N. V. Giai, Nucl. Phys. A 731, 15 (2004).

[98] D. Vretenar, T. Niksic, and P. Ring, Phys. Rev. C 68, 024310 (2003).

[99] J. Piekarewicz and M. Centelles, Phys. Rev. C 79, 054311 (2009).

[100] J. P. Blaizot, J. F. Berger, J. Dechargé, and M. Girod, Nucl. Phys. A 591, 435 (1995).

[101] E. Khan, J. Margueron, and I. Vidaña, Phys. Rev. Lett. 109, 092501 (2012).

[102] V. B. Soubbotin, V. I. Tselyaev, and X. Viñas, Phys. Rev. C 69, 064312 (2004).

[103] S. K. Patra, X. Viñas, M. Centelles, and M. Del Estal, Nucl. Phys. A 703, 240 (2002).

[104] Y.-W. Lui, D. H. Youngblood, Y. Tokimoto, H. L. Clark, and B. John, Phys. Rev. C 69, 034611 (2004).

[105] F. A. Brieva and J. R. Rook, Nucl. Phys. A 297, 206 (1978).

[106] M. Kohno, Phys. Rev. C 86, 061301 (2012).

[107] J. Dukelsky, S. Lerma H., L. M. Robledo, R. RodriguezGuzman, and S. M. A. Rombouts, Phys. Rev. C 84, 061301 (2011).

[108] S. K. Patra, M. Centelles, X. Viñas, and M. Del Estal, Phys. Rev. C 65, 044304 (2002).

[109] L. W. Chen, B. J. Cai, C. M. Ko, Bao-an Li, C. Shen, and J. Xu, Phys. Rev. C 80, 014322 (2009).

[110] M. Centelles, X. Viñas, S. K. Patra, J. N. De, and Tapas Sil, Phys. Rev. C 72, 014304 (2005). 